\title{
¿Cuánto cuesta la densificación? La relación entre la densidad y el costo de proveer servicios urbanos básicos en Brasil, Chile, Ecuador y México
}

Nora Libertun. Banco Interamericano de Desarrollo, Washington D.C., Estados Unidos.

Roberto Guerrero. Banco Interamericano de Desarrollo, Washington D.c., Estados Unidos.

RESUMEN | La relación entre el gasto municipal per cápita en servicios urbanos básicos y la densidad de población urbana no es lineal, y alcanza niveles de gastos óptimos en densidades cercanas a 9.000 habitantes por kilómetro cuadrado. El 85\% de los municipios de Brasil, Chile, Ecuador y México están por debajo de este nivel ideal de densidad. Estos hallazgos indican que las políticas que promueven la urbanización y densificación de las ciudades intermedias pueden tener importantes beneficios respecto a la reducción de los gastos municipales.

PALABRAS ClAVE | urbanización, servicios urbanos, ciudades intermedias.

ABSTRACT | The relationship between per capita municipal spending on basic urban services and urban population density is not a linear one. The optimal density level that minimizes spending in urban services is close to 9,000 inhabitants per square kilometer. In Brazil, Chile, Ecuador and Mexico, 85\% of the municipalities find themselves below the ideal density level. The findings of this article suggest that policies that promote urbanization and densification of intermediate cities can have significant benefits in reducing municipal expenditures.

KEYWORDs | urbanization, urban services, intermediate cities. 


\section{Introducción}

Las ciudades densas son una opción racional para un mundo cada vez más urbanizado, donde las preocupaciones acerca de la sostenibilidad ambiental y la expansión urbana son primordiales (UN-Habitat, 2012). Entre sus muchas ventajas, las ciudades densas ayudan a preservar las tierras fértiles para usos rurales (Jenks \& Burgess, 2000), disminuir los tiempos dedicados a traslados (Gaigne, Riou \& Thisse, 2012) y contribuyen a reducir las emisiones de gases de efecto invernadero (Stone, Mednick, Holloway \& Spak, 2007). Además, la densidad se correlaciona positivamente con la acumulación de capital humano (Glaeser, 1999), la tasa de innovación (Carlino, Chatterjee \& Hunt, 2007), la productividad del trabajo (Ciccone \& Hall, 1996) y la inclusión social (Burton, 2000), siendo la aglomeración de actividades económicas en el territorio un factor determinante de la formación de concentraciones humanas (Krugman, 1991). En general, las áreas de mayor desarrollo económico están representadas como un sistema de economías polarizadas vinculadas a una aglomeración central (Scott, 2006). Por estos motivos, y en la búsqueda de integrar las políticas de desarrollo económico con objetivos ambientales y sociales, se promueve la densificación urbana en países tanto desarrollados como en vías de desarrollo. Las Naciones Unidas (un-Habitat, 2012), el Banco Mundial (World Bank, 2014a), la Organización para la Cooperación y el Desarrollo Económicos (oECD, 2012) y el Banco Interamericano de Desarrollo (Inter-American Development Bank [IADB], 2013), entre otros, apoyan la densificación urbana. Los planes de desarrollo de varios países, como por ejemplo México (2013), Colombia (2011), China (2011) y Sudáfrica (2012), también abogan por una mayor densidad urbana. Incluso los planes de desarrollo de ciudades consideradas densas, como Londres (2013), Nueva York (2011) y Monterrey (2011), aplican políticas explícitas para aumentar su densidad.

No obstante, mantener poblaciones densas tiene sus costos. La densidad urbana aumenta los precios de la tierra (Glaeser, Kolko \& Saiz, 2001), de los salarios (Wheaton \& Lewis, 2001), de la congestión (Wheaton, 1998), e incrementa la tasa de criminalidad (Glaeser \& Sacerdote, 1999). Aún no es claro su impacto en el gasto público. Algunos estudios muestran que la densidad se relaciona con ahorros en la protección contra incendios, recolección de residuos y servicios de educación (Bollinger, Berger \& Thompson, 2001). Pero otros correlacionan la densidad con deseconomías de escala para esos mismos servicios (Abrate, Erbetta, Fraquelli \& Vannoni, 2012). Incluso en otros estudios, no se atribuye ningún impacto a las altas densidades sobre el gasto en protección contra incendios y recolección de residuos sólidos; pero se indica que sí contribuyen a reducir los gastos en servicios de policía y educación y en la construcción de nueva infraestructura y carreteras (Carruthers \& Ulfarsson, 2003, 2008). Por último, otros proponen una relación en forma de $U$ entre densidad y gasto, sugiriendo que pasada cierta densidad óptima, los gastos aumentan (Holcombe \& Williams, 2008; Ladd, 1992).

Además, la densidad de la población puede ser endógena al gasto en servicios público, lo cual la actual literatura desconoce al asumir que el gasto en servicios es función lineal de la densidad. Bajo condiciones favorables, el proceso de aglomeración poblacional incrementa la demanda por servicios básicos, cuya provisión 
es financiada por los gobiernos locales. Sin embargo, también es posible el caso inverso, y que los residentes se muden a aquellos municipios que ofrecen servicios urbanos básicos, puesto que una mayor oferta de servicios se traduce en niveles más altos de bienestar social y una calidad de vida más elevada, incentivando a poblaciones rurales a emigrar a zonas urbanas, densificándolas en el proceso. La historia urbana de América Latina ofrece ejemplos específicos para ambos escenarios: la densificación genera inversiones en infraestructura de servicios urbanos básicos, como en los programas de mejoramiento de barrios en Brasil (Brakarz, Greene \& Rojas, 2002). Por el contrario, las inversiones en infraestructura de servicios urbanos básicos también generan densificación, con Brasilia como el ejemplo canónico de las ciudades planificadas. Asimismo, la cobertura de los servicios urbanos básicos puede ser incompleta, lo cual es el caso en los países en desarrollo. Por ejemplo, cerca de un tercio de la población urbana de América Latina carece de algún servicios urbano básico (Bouillon, 2012), siendo este un desafío para los pequeños gobiernos municipales recientemente incluidos en la expansión de las áreas metropolitanas (Campbell, 2012). Y, sin embargo, la inmigración urbana no merma (Feler \& Henderson, 2011). Si nos guiamos por la historia, la falta de servicios urbanos básicos no frena el crecimiento de la población urbanizada de América Latina, sino más bien propicia la provisión de estos servicios mediante arreglos informales.

La urbanización de América Latina expone la endogeneidad y falta de universalidad en la cobertura de los servicios urbanos básicos. Entre 1960 y 2010, la proporción de la población urbana de América Latina aumentó de 50\% a 80\%, una tasa de urbanización más alta que la de Europa e igual a la de Estados Unidos (World Bank, 2014b). La cantidad de ciudades densas también aumentó notablemente: mientras que en 1950 el número de ciudades con más de 500.000 habitantes era 12, hoy es 125. Como era de esperarse, este crecimiento vino acompañado por un aumento en la demanda de servicios urbanos básicos y en el número de municipios responsables por su prestación (BID, 2013). Sin embargo, la capacidad fiscal de los municipios no ha crecido lo suficiente (Bonet, De la Cruz \& Fretes, 2013), llevando a una urbanización con graves déficits. Hoy en día, la brecha de prestación de servicios básicos es considerable: más de 13 millones de residentes urbanos carecen de acceso a fuentes mejoradas de agua, y 64 millones carecen de servicios de saneamiento en sus viviendas (World Bank, 2014b). Cerrar la brecha de la demanda de servicios de agua para el año 2030 tendrá un costo de más de Us\$ 100 mil millones, mientras que se necesitan otros us\$ 79 mil millones para cerrar el déficit de saneamiento actual (Corporación Andina de Fomento [CAF], 2013).

En este contexto, cabe preguntarse cómo los patrones de crecimiento urbano actuales impactan en los costos de provisión de servicios urbanos básicos. ¿Acaso los municipios más densos tienen una mejor cobertura de servicios urbanos básicos? ¿Cómo impacta la densidad de un municipio en su gasto per cápita en servicios urbanos básicos? La contribución principal de este estudio es responder a estas preguntas para América Latina, tomando en consideración que la cobertura de servicios urbanos básicos actual es incompleta —el 100\% del gasto se distribuye en menos del $100 \%$ de la población - y que existe una condición de endogeneidad entre la densidad de población y el gasto en servicios. En el estudio, modelamos 
el gasto de servicios urbanos básicos en función de su demanda y de sus costos, teniendo en cuenta los niveles de cobertura reales; e incorporamos datos climáticos como variables instrumentales para establecer el efecto causal del aumento de la densidad sobre el gasto municipal. Además, utilizamos datos de países en desarrollo, donde estos temas aún no han sido estudiados en profundidad.

Los datos de este estudio constan de un panel de aproximadamente 17.000 observaciones, referidas a 8.585 municipios de Brasil, Chile, Ecuador y México, en los ańos 2000 y 2010. La información estadística respectiva a la década anterior (1990) presenta deficiencias en su recopilación y no se incluyó en el presente estudio. Consideramos tres servicios urbanos básicos cuya prestación es organizada y financiada por los gobiernos municipales: agua, alcantarillado y recolección de residuos. El análisis incorpora únicamente estos servicios por tres razones. En primer lugar, la disponibilidad de información con suficiente comparabilidad entre países; otros servicios urbanos, como áreas verdes y cuidados de espacios públicos, no cuentan con información estadística desagregada que permita realizar comparaciones confiables. Segundo, otros servicios, tales como la educación y la salud, suelen ser provistos por distintos niveles de gobierno, el sector privado, o directamente por el propio hogar. Finalmente, estos tres servicios son, en términos de las finanzas públicas municipales, de relevancia considerable. En los países considerados en este estudio, los municipios gastan aproximadamente una séptima parte de su presupuesto en ellos (véase gráfico $\mathrm{C}$ en la tabla 1). La diversidad de estos países proporciona una buena base para el modelo aplicado en este trabajo. México y Brasil son grandes países federales; Chile y Ecuador son pequeños y centralizados. Las tasas de urbanización de estos países también son diferentes: 68\% en Ecuador, $78 \%$ en México, y 85\% en Brasil y 89\% en Chile. Aproximadamente el 28\% de los residentes urbanos brasileńos vive en asentamientos informales que carecen de algún servicio básico; esta cifra es del $21 \%$ en Ecuador, 14\% en México y $9 \%$ en Chile. El producto bruto interno (PBI) medio per cápita en 2010 fue de US\$ 8.916 en México; us\$12.685 en Chile, us\$ 10.978 en Brasil y us\$ 4.637 en Ecuador (World Bank, 2014b). Cabe destacar que la población combinada de estos cuatro países equivale a casi el $60 \%$ de la población total de América Latina y el Caribe.

TABLA I | Estadísticas descriptivas

\begin{tabular}{|l|c|c|c|}
\hline & MEDIA & \multicolumn{1}{|c|}{$\begin{array}{c}\text { DESVIACIÓN } \\
\text { ESTÁNDAR }\end{array}$} & $\begin{array}{c}\text { NÚMERO DE } \\
\text { OBSERVACIONES }\end{array}$ \\
\hline & A. CARACTERÍSTICAS DEMOGRÁFICAS (2000-20IO) \\
\hline Densidad de población total (personas $/ \mathrm{km}^{2}$ ) & 176 & 919,20 & 17.092 \\
\hline Densidad de población urbana (personas $/ \mathrm{km}^{2}$ ) & 13.126 & $85.583,85$ & 15.439 \\
\hline $\begin{array}{l}\text { Densidad de población urbana con cobertura } \\
\left.\text { (personas } / \mathrm{km}^{2}\right)\end{array}$ & 8.447 & $47.152,56$ & 14.795 \\
\hline Población total & 36.574 & 174.349 & 17.096 \\
\hline Tasa de población urbana & 54 & 29 & 17.092 \\
\hline Tamańo promedio de hogar & 3,89 & 0,65 & 17.082 \\
\hline Tasa de pobreza & 0,37 & 0,24 & 17.105 \\
\hline
\end{tabular}


(continuación)

\begin{tabular}{|c|c|c|c|}
\hline & MEDIA & $\begin{array}{l}\text { DESVIACIÓN } \\
\text { ESTÁNDAR }\end{array}$ & $\begin{array}{l}\text { NÚMERO DE } \\
\text { OBSERVACIONES }\end{array}$ \\
\hline & \multicolumn{3}{|c|}{ B. ACCESO A SERVICIOS MUNICIPALES (2000-20IO) } \\
\hline $\begin{array}{l}\text { Cobertura de servicios-( } 3 \text { servicios, alta calidad }) \\
\text { (total) }\end{array}$ & 0,51 & 0,24 & 17.087 \\
\hline $\begin{array}{l}\text { Cobertura de servicios-( } 3 \text { servicios, alta calidad }) \\
\text { en población urbana }\end{array}$ & 0,69 & 0,21 & 15.268 \\
\hline Cobertura de servicios de agua (total) & 0,67 & 0,24 & 17.087 \\
\hline $\begin{array}{l}\text { Cobertura de servicios de agua (población } \\
\text { urbana) }\end{array}$ & 0,87 & 0,19 & 15.268 \\
\hline Cobertura de servicios de saneamiento (total) & 0,31 & 0,31 & 17.087 \\
\hline $\begin{array}{l}\text { Cobertura de servicios de saneamiento (pobla- } \\
\text { ción urbana) }\end{array}$ & 0,44 & 0,38 & 15.268 \\
\hline $\begin{array}{l}\text { Cobertura de servicios de recolección de residuos } \\
\text { (total) }\end{array}$ & 0,55 & 0,28 & 11.507 \\
\hline \multirow[t]{2}{*}{$\begin{array}{l}\text { Cobertura de servicios de recolección de residuos } \\
\text { (población urbana) }\end{array}$} & 0,77 & 0,28 & 11.506 \\
\hline & \multicolumn{3}{|c|}{$\begin{array}{l}\text { C. CARACTERÍSTICAS FISCALES Y ECONÓMICAS } \\
(2000-2010) \text { US } \$\end{array}$} \\
\hline Gasto municipal & 18,51 & 169,22 & 16.731 \\
\hline Gasto municipal en servicios & 2,61 & 17,70 & 16.710 \\
\hline Agua & 0,76 & 5,69 & 16.210 \\
\hline Saneamiento & 0,70 & 6,74 & 11.130 \\
\hline Recolección de basura & 0,88 & 9,97 & 16.210 \\
\hline Ingresos fiscales & 3,08 & 69,56 & 16.738 \\
\hline Ingresos fiscales inmobiliarios & 1,15 & 23,95 & 15.287 \\
\hline Transferencias intergubernamentales & 12,49 & 73,00 & 16.738 \\
\hline Salario promedio & 4.710 & 3.211 & 16.785 \\
\hline Tasa de desempleo & 0,07 & 0,06 & 16.814 \\
\hline \multirow[t]{2}{*}{ Ingresos } & 5.089 & 5.733 & 17.014 \\
\hline & \multicolumn{3}{|c|}{ D. CARACTERÍSTICAS MUNICIPALES (2000-20IO) } \\
\hline Extensión total $\left(\mathrm{km}^{2}\right)$ & 1.484 & 14.303 & 17.168 \\
\hline Extensión área urbanizada $\left(\mathrm{km}^{2}\right)$ & 3 & 12 & 17.020 \\
\hline Pertenencia a área metropolitana & 0,11 & 0,31 & 17.172 \\
\hline \multirow[t]{2}{*}{ Cabecera urbana } & 0,05 & 0,22 & 16.588 \\
\hline & \multicolumn{3}{|c|}{ E. INDICADORES CLIMÁTICOS (I9IO-I930 Y 20I2) } \\
\hline Temperatura anual promedio, rezagada $\left({ }^{\circ} \mathrm{C}\right)$ & 21,0 & 4,3 & 17.042 \\
\hline Humedad del suelo, rezagada (Índice Palmer) & 0,2 & 1,0 & 16.239 \\
\hline Precipitaciones anuales, rezagada $(\mathrm{mm})$ & $1.197,0$ & 490,1 & 17.042 \\
\hline Temperatura anual promedio, actual $\left({ }^{\circ} \mathrm{C}\right)$ & 22,1 & 5,2 & 17.042 \\
\hline Precipitaciones anuales, actual (mm) & $1.102,5$ & 870,9 & 17.042 \\
\hline
\end{tabular}

FUENTE ELABORACIÓN PROPIA 


\section{La densidad de población y el costo de los servicios urbanos básicos}

Mientras los municipios intentan cerrar la brecha de servicios urbanos básicos, es crítico saber cómo los patrones de crecimiento urbano incidirán en el costo de proveer dichos servicios. La densidad de población es uno de los indicadores claves de la distribución espacial de los residentes, y el más utilizado en la literatura (Forsyth, 2003). Su prominencia en los estudios empíricos indica que a pesar de sus deficiencias vis$\grave{a}$-vis dimensiones especiales matizadas, tales como la continuidad, la nuclearidad y la centralidad, es útil para los trabajos comparativos urbanos (Angel, Sheppard \& Civco, 2005). Mientras que otras dimensiones espaciales revelan con mayor especificidad la dinámica de crecimiento de una ciudad, la densidad permite analizar y comparar la urbanización de un modo menos propenso a errores de interpretación y más intuitivo (Rapoport, 1977). Además, al utilizar la densidad, podemos armonizar los hallazgos de este trabajo con los de aquellos que estudian la urbanización respecto a su desempeño económico (Henderson, 2003) y con el lenguaje de las recomendaciones de política públicas. Por otra parte, en un contexto social democrático, los instrumentos para la gestión de la densidad urbana son más fáciles de implementar que aquellos que buscan limitar el crecimiento de la población urbana.

Dado que la densidad de población es relevante para muchos aspectos de la urbanización, incluyendo su desarrollo social y económico, podría suponerse que está claro su impacto en las finanzas públicas locales. Sin embargo, no es así. El impacto de la densidad de población en los patrones de gasto local, aunque ampliamente estudiado y documentado, es objeto de controversia empírica. Las conclusiones de la literatura sobre los efectos de la densidad son —irónicamente- notoriamente disgregadas, con claras discrepancias tanto en términos de magnitud como de signo. Aunque existe consenso respecto a la idea de un nivel de densidad óptimo para la provisión y prestación de servicios urbanos básicos, los estudios difieren respecto de cuál es ese nivel óptimo.

Quienes argumentan que la densidad de población disminuye el costo per cápita de la prestación de servicios, suponen que esta genera economías de escala. Desde esta perspectiva, el crecimiento urbano de baja densidad requiere expandir la provisión de infraestructura a lugares poco poblados, disminuyendo así los costos per cápita. Coyne (2003) muestra que entre 1980 y 2000, las políticas de densificación en Colorado, Estados Unidos, llevaron a un aumento del $27 \%$ en la densidad de población y a una reducción del $7 \%$ en el gasto per cápita. Esto coincide con un informe para la ciudad de Calgary, Canadá (Іві Group, 2009), según el cual un aumento del $25 \%$ de la densidad reduciría el gasto público en la provisión de carreteras, protección contra el fuego y el agua en un 36\%, 46\% y 54\%, respectivamente. Esto se asemeja a otros resultados para condados de Estados Unidos. Burchell y Mukherji (2003) encuentran que densificar en un 11\% los hogares existentes disminuye en $7 \%$ los costos de la infraestructura de agua y alcantarillado, 12\% los de carreteras y $8 \%$ los de vivienda. Carruthers y Ulfarsson (2003) muestran que aumentar la densidad en un $1 \%$ disminuye en $4,2 \%$ el costo de servicios de policía y de educación, y en $3 \%$ el costo combinado de doce servicios urbanos. 
Por otra parte, varios autores sostienen que la densidad no necesariamente se asocia a economías de escala. En España, Hortas-Rico y Sole-Olle (2010) encuentran que duplicar el área urbanizada aumenta el costo de las instalaciones comunitarias en un $11 \%$, de la policía local en un $9 \%$, de vivienda en un $8 \%$, de servicios recreativos en un $15 \%$ y de gastos administrativos en un $11 \%$. En un estudio sobre los condados de Estados Unidos, Pineda (2005) indica que los servicios urbanos de mano de obra (por ejemplo, policía, protección contra incendios, salud) aumentan sus costos per cápita cuando se incrementa la densidad de población. Ladd y Yinger (1989) demuestran que una alta densidad media eleva los costos de los servicios públicos debido a un "entorno más demandante". Cameron (1989) encuentra que una mayor densidad implica mayores costos para los servicios de policía. Holcombe y Williams $(2008,2010)$ señalan que en los municipios de más de 500.000 habitantes de Florida, una mayor densidad de población se asocia a más gastos per cápita, en particular para alcantarillado, policía y carreteras. Carruthers y Ulfarsson (2003) establecen que si se excluyen las carreteras, los costos de transporte aumentan con la densidad.

Un tercer punto de vista es que, inicialmente, la densificación genera ahorros en los costos de los servicios públicos, pero que ellos se disipan conforme la densidad aumenta. Esto sugiere una relación en forma de $U$ entre densidad y gasto urbano, con un nivel óptimo de densidad. Werner Hirsch (1959) realizó uno de los primeros análisis empíricos en apoyo de esta teoría, basándose en los datos de protección contra incendios de los condados de Estados Unidos. Ladd (1992) demuestra que la función de gastos de los condados semeja una parábola cuyo vértice está en 250 habitantes por milla cuadrada; encuentra que el gasto promedio per cápita de los condados de muy baja y muy alta densidad (hasta 125 y más de 24.000 habitantes por milla cuadrada) es $14 \%$ y $43 \%$ más alto que en los municipios en un rango óptimo de densidad. En España, Álvarez, Prieto y Zofio (2013) muestran que los niveles óptimos de densidad varían para cada servicio prestado, con valores en habitantes por kilómetro cuadrado que van desde 2.800 para la pavimentación y la iluminación, 3.100 habitantes para la provisión de agua, y 4.400 para el alcantarillado.

En resumen, la relación entre densidad de población y gasto público per cápita es significativa y, sin embargo, su dinámica es ambigua (Boyko \& Cooper, 2011; Ewing, 1994). En el mejor de los casos, esta inconsistencia empírica puede deberse a diferentes definiciones de datos y unidades de análisis. Una preocupación más grave es que se deba a que los modelos utilizados no consideran apropiadamente la existencia de variables explicativas, resultando en una falta de identificación adecuada. Lo que es claro es que dos retos permanecen. El primero es empírico. La falta de datos en países menos desarrollados ha restringido los estudios a aquellos donde hay datos confiables a nivel local. Pero la falta de investigación empírica sobre los patrones de urbanización en los países en desarrollo lleva a recomendaciones de políticas mal informadas (Angel et al., 2005). Los modelos utilizados carecen de variables tales como porcentaje de hogares urbanos sin acceso a servicios básicos, la cual refleja una dinámica específica del proceso de urbanización en estos países (Libertun de Duren, 2011). El segundo reto es metodológico. La literatura no considera la determinación endógena entre gastos municipales y densificación, 
estableciendo simultáneamente los valores de densidad y de gastos, sin iluminar las dinámicas del crecimiento. En el caso de los países en desarrollo, donde suele existir un rezago considerable entre el asentamiento de la población y la provisión de servicios, esta indeterminación puede llevar a conclusiones erradas.

\section{Metodología}

En este estudio, consideramos hogares con cobertura aquellos que reciben servicios urbanos básicos de alta calidad. El supuesto es que los hogares informales y rurales tienen más probabilidades de acceder a servicios mediante otras modalidades. Los hogares urbanos informales a menudo dependen de camiones de agua, alcantarillados abiertos y contenedores comunales de residuos de basura; mientras que los rurales utilizan pozos de agua, cámaras sépticas individuales y contenedores individuales de residuos (World Bank, 2014b). Además, considerar una única modalidad de cobertura mejora la comparabilidad de las estimaciones a la hora de evaluar el gasto público. Por último, desde un punto de vista normativo, los servicios urbanos básicos de alta calidad son lo que se espera para las áreas urbanas.

En el modelo, el gasto municipal en servicios urbanos básicos es función de los gastos en provisión de servicios — agua, saneamiento y recolección de residuos- y de la demanda de los hogares. Asumimos que el costo de producción de estos servicios $(C)$ depende de un índice de costos $(w)$ y de ser cabecera urbana $(m)$, ya que estas unidades actúan como puntos focales para la prestación de servicios urbanos básicos (ecuación 1). El nivel de cobertura (c) depende de los fondos municipales para proporcionar dicha cobertura $(e)$, dividido por la densidad de población $(d), y$ otros factores de costo $(z)$; suponiendo rendimientos constantes a escala (ecuación 2):

$$
\begin{aligned}
& C=c \cdot f(d) \cdot g(z) \cdot w m \\
& c=\frac{e}{f(d) \cdot g(z)}
\end{aligned}
$$

Combinamos esta función de costo con un modelo de demanda que maximiza la utilidad de los residentes municipales; la demanda de cobertura disminuye según su parte del costo marginal de proveer dicha cobertura $(c)$. La restricción presupuestaria de un residente $\left(y_{r}\right)$ depende de cuantos servicios consume $\left(x_{r}\right)$, de la tasa del impuesto municipal $(t)$ y la tasa de impuestos individual $\left(b_{r}\right)$ (ecuación 3). La restricción presupuestaria del gobierno municipal $(C)$ depende de su base impositiva $(B)$ y de las transferencias intergubernamentales que recibe $(G)$ (ecuación 4). La ecuación 5 indica que un gobierno municipal que maximiza la utilidad de los residentes está limitado por las restricciones presupuestarias propias $(C)$ y de los residentes $\left(y_{r}\right)$ y por la función de costo de la cobertura de servicios urbanos básicos (ecuación 2):

$$
y_{r}=x_{r}+t b_{r}
$$


$C=t B+G$

$x_{r}+c \cdot f(d) \cdot g(z) \cdot w m \cdot \frac{b_{r}}{b}=y_{r}+t \cdot \frac{b_{r}}{b}$

Si maximizamos la función de utilidad (ecuación 3), obtenemos la condición de primer orden (ecuación 6), en la cual el precio de los impuestos $\left(p_{r}\right)$ es el producto del costo marginal de la cobertura de los servicios urbanos básicos por la cuota tributaria. Suponiendo que la función de demanda es log-lineal (ecuación 7), y sustituyendo la ecuación (6) en la ecuación (7) y la formulación resultante en la ecuación (2), obtenemos la función log gasto (ecuación 8):

$$
\begin{aligned}
& \frac{\partial U_{r} / \partial c}{\partial U_{r} / \partial x_{r}}=f(d) \cdot g(z) \cdot w m \cdot \frac{b_{r}}{b} \equiv p_{r} \\
& c=k \cdot p_{r}^{\alpha} \cdot\left(y_{r}+g \cdot \frac{b_{r}}{b}\right)^{\beta} \cdot v_{r}^{\gamma} \\
& \ln \varphi=\ln k+(\alpha+1) \cdot \ln (f(d))+(\alpha+1) \cdot \ln g(z)+(\alpha+1) \cdot \ln w+(\alpha+1) \cdot \ln m+\alpha \ln \left(\frac{b_{r}}{b}\right)+ \\
& \beta \ln y_{r}+\beta \cdot\left(\frac{g}{y_{r}}\right)\left(\frac{b_{r}}{b}\right)+\gamma \cdot \ln v_{r}
\end{aligned}
$$

La última ecuación (8) evidencia que el gasto per cápita en servicios urbanos básicos de un municipio depende del costo de proporcionar dichos servicios, de la densidad de la población con cobertura $(f(d))$ y de otras características propias del municipio. Estas últimas incluyen el número potencial de usuarios de los servicios, el tamaño medio del hogar, el porcentaje de hogares urbanos, el porcentaje de hogares desempleados y el salario promedio municipal $(w)$. Además, de si el municipio pertenece un área metropolitana o es cabecera regional.

La ecuación (8) también refleja que la demanda de servicios urbanos básicos determina el gasto municipal. Por lo tanto, incluye ingresos $\left(y_{r}\right)$, cuota tributaria típica $\left(b_{r} / b\right)$ e impuesto local per cápita. Este último se define como el impuesto a la propiedad per cápita dividido por los ingresos tributarios per cápita. El precio del impuesto equivale al producto del costo marginal de la cobertura por los ingresos fiscales del municipio. Incluimos también las transferencias intergubernamentales en relación con los ingresos propios $\left(g / y_{r}\right)$, para considerar el efecto de las transferencias en los ingresos municipales. Por último, la tasa de pobreza municipal $(v)$, en tanto el nivel de ingresos, determina la demanda de los hogares (Gilens, 2009).

Otras variables, tales como los precios locales y la geografía, pueden afectar el gasto local, por lo que no necesariamente un mayor gasto implica más servicios urbanos básicos. Para evitar confundir la influencia de estos factores con la densidad, incluimos efectos fijos municipales para controlar las diferencias entre municipios que pueden influir en el gasto municipal, y efectos fijos irrestrictos de tiempo para controlar las variaciones temporales en el gasto público municipal. Por supuesto, incluimos también un término de error con las propiedades típicas.

Finalmente, utilizamos una fuente exógena de variación para resolver la determinación simultánea de la densidad y gasto público. Siguiendo a Rosenthal y Strange (2003), Combes, Duranton, Gobillon y Roux, (2010), y Glaeser y Gottlieb (2009), 
empleamos variables climáticas como instrumentos de control. Nuestra estrategia se basa en la identificación de la ortogonalidad de estas variables a los cambios en los gastos de servicios urbanos básicos a nivel municipal, excepto cuando los cambios en el nivel de gastos se deben a la densidad de población. Aunque en las zonas rurales el clima tiene un efecto directo sobre los ingresos (Guerrero Compeán, 2013), nuestra estrategia de identificación es apropiada para las áreas urbanas, ya que el clima es un factor determinante de los patrones de asentamiento, pero no está fuertemente vinculado al crecimiento de los ingresos de la población (Miguel, Satyanath \& Sergenti, 2004).

\section{Datos, estrategias de recolección y limitaciones}

Los datos de población, cobertura y calidad de servicios urbanos básicos, y características urbanas de cada municipio, están basados en los censos nacionales; los datos fiscales surgen de las bases de datos de cuentas municipales compiladas a nivel nacional; y los indicadores climáticos, de la base de datos de la Universidad de East Anglia (UEA) y la Administración Oceánica y Atmosférica Nacional (NOAA). Luego de resolver conflictos semánticos y armonizar conceptos, conformamos un panel único que incluye los datos de los cuatro países.

TABLA 2 Datos y fuentes, por país

\begin{tabular}{|c|c|c|c|c|}
\hline $\begin{array}{l}\text { DATOS A NIVEL } \\
\text { MUNICIPAL }\end{array}$ & BRASIL & CHILE & ECUADOR & MÉXICO \\
\hline \multicolumn{5}{|c|}{ Características demográficas $(2000,2010)$} \\
\hline $\begin{array}{l}\text { Número de residentes; de } \\
\text { residentes pobres; de resi- } \\
\text { dentes urbanos }\end{array}$ & \multirow{2}{*}{$\begin{array}{l}\text { IBGE Sistema } \\
\text { de Recuperaçấo } \\
\text { Automática. }\end{array}$} & \multirow{2}{*}{$\begin{array}{l}\text { INE XVII Censo } \\
\text { Nacional de } \\
\text { Población y VI } \\
\text { de Vivienda. }\end{array}$} & \multirow{2}{*}{$\begin{array}{l}\text { INEC XVII } \\
\text { Censo Nacional } \\
\text { de Población y VI } \\
\text { de Vivienda. }\end{array}$} & \multirow{2}{*}{$\begin{array}{l}\text { INEGI Censo } \\
\text { General de } \\
\text { Población y } \\
\text { Vivienda. }\end{array}$} \\
\hline $\begin{array}{l}\text { Tamańo medio de hogar por } \\
\text { municipio }\end{array}$ & & & & \\
\hline \multicolumn{5}{|c|}{ Acceso a servicios municipales $(2000,2010)$} \\
\hline $\begin{array}{l}\text { Número de hogares con gri- } \\
\text { fo en la vivienda conectado } \\
\text { al servicio por tubería; con } \\
\text { sistema de alcantarillado } \\
\text { entubado; con recolección } \\
\text { domiciliaria de residuos }\end{array}$ & $\begin{array}{l}\text { IBGE Sistema } \\
\text { de Recuperaçáo } \\
\text { Automática. }\end{array}$ & $\begin{array}{l}\text { INE XVII Censo } \\
\text { Nacional de } \\
\text { Población y VI } \\
\text { de Vivienda. }\end{array}$ & $\begin{array}{l}\text { INEC XVII } \\
\text { Censo Nacional } \\
\text { de Población y VI } \\
\text { de Vivienda. }\end{array}$ & $\begin{array}{l}\text { INEGI Censo } \\
\text { General de } \\
\text { Población y } \\
\text { Vivienda. }\end{array}$ \\
\hline \multicolumn{5}{|c|}{ Características fiscales y económicas $(2000,2010)$} \\
\hline $\begin{array}{l}\text { Ingresos fiscales totales; } \\
\text { ingresos por impuesto } \\
\text { inmobiliario }\end{array}$ & $\begin{array}{l}\text { Tesouro Nacio- } \\
\text { nal. Finanças do } \\
\text { Brasil. Dados } \\
\text { Contábeis dos } \\
\text { Municípios. }\end{array}$ & \multirow{3}{*}{$\begin{array}{l}\text { SUBDERE. } \\
\text { Sistema Nacional } \\
\text { de Información } \\
\text { Municipal. }\end{array}$} & \multirow{3}{*}{$\begin{array}{l}\text { SENPLADES. } \\
\text { Ingresos y gastos } \\
\text { del sector público } \\
\text { a nivel cantonal. }\end{array}$} & \multirow{3}{*}{$\begin{array}{l}\text { INEGI. Estadís- } \\
\text { tica de finanzas } \\
\text { públicas estatales } \\
\text { y municipales. }\end{array}$} \\
\hline $\begin{array}{l}\text { Ingresos por transferencias } \\
\text { intergubernamentales }\end{array}$ & \multirow{2}{*}{$\begin{array}{l}\text { IBGE Sistema } \\
\text { de Recuperação } \\
\text { Automática. }\end{array}$} & & & \\
\hline $\begin{array}{l}\text { Gastos totales; gastos en } \\
\text { servicios públicos }\end{array}$ & & & & \\
\hline
\end{tabular}


(continuación)

\begin{tabular}{|c|c|c|c|c|}
\hline $\begin{array}{l}\text { DATOS A NIVEL } \\
\text { MUNICIPAL }\end{array}$ & BRASIL & CHILE & ECUADOR & MÉXICO \\
\hline $\begin{array}{l}\text { Compensación empleados } \\
\text { municipales }\end{array}$ & \multirow{4}{*}{$\begin{array}{l}\text { IBGE Sistema } \\
\text { de Recuperação } \\
\text { Automática. }\end{array}$} & $\begin{array}{l}\text { MIDEPLAN. } \\
\text { Encuesta de } \\
\text { Caracterización } \\
\text { Socioeconómica } \\
\text { Nacional 2003, } \\
2009 .\end{array}$ & \multirow[t]{2}{*}{$\begin{array}{l}\text { INEC XVII } \\
\text { Censo Nacional } \\
\text { de Población y VI } \\
\text { de Vivienda. }\end{array}$} & \multirow[t]{2}{*}{$\begin{array}{l}\text { INEGI. Censos } \\
\text { Económicos } \\
1999,2009 .\end{array}$} \\
\hline Salario promedio & & \multirow{4}{*}{$\begin{array}{l}\text { MINSAL. Base } \\
\text { de datos del país } \\
\text { a nivel comuna } \\
2009,2011 \\
\text { Observatorio So- } \\
\text { cial, Ministerio } \\
\text { de Desarrollo } \\
\text { Social. Pobreza } \\
\text { por comunas } \\
2003,2009 .\end{array}$} & & \\
\hline Producto Bruto Interno & & & $\begin{array}{l}\text { Banco Central } \\
\text { del Ecuador. } \\
\text { Valor agregado } \\
\text { bruto cantonal } \\
2007 \text { y Cuentas } \\
\text { Provinciales } \\
1999 .\end{array}$ & $\begin{array}{l}\text { CONEVAL. } \\
\text { ICTPC anual } \\
2010 .\end{array}$ \\
\hline Tasa de desempleo & & & \multirow{2}{*}{$\begin{array}{l}\text { INEC XVII } \\
\text { Censo Nacional } \\
\text { de Población y VI } \\
\text { de Vivienda. }\end{array}$} & $\begin{array}{l}\text { INEGI Censo } \\
\text { General de } \\
\text { Población y } \\
\text { Vivienda. }\end{array}$ \\
\hline Tasa de pobreza & $\begin{array}{l}\text { UNDP Brazil. } \\
\text { Atlas do De- } \\
\text { senvolvimento } \\
\text { Humano } 2013 .\end{array}$ & & & $\begin{array}{l}\text { CONEVAL. } \\
\text { Estimaciones de } \\
\text { pobreza alimen- } \\
\text { taria. }\end{array}$ \\
\hline Índice de precios & $\begin{array}{l}\text { IBGE Sistema } \\
\text { de Recuperação } \\
\text { Automática. }\end{array}$ & $\begin{array}{l}\text { INE. Estadísticas } \\
\text { de Precio. }\end{array}$ & $\begin{array}{l}\text { INEC. Índice de } \\
\text { Precios al Consu- } \\
\text { midor. }\end{array}$ & $\begin{array}{l}\text { Banco de } \\
\text { México. Índices } \\
\text { de precios al } \\
\text { consumidor. }\end{array}$ \\
\hline \multicolumn{5}{|c|}{ Indicadores urbanos $(2000,2010)$} \\
\hline Coordenadas geográficas & \multirow{2}{*}{$\begin{array}{l}\text { UNDP Brazil } \\
\text { Caracterização } \\
\text { do Município. }\end{array}$} & \multirow{2}{*}{$\begin{array}{l}\text { Instituto } \\
\text { Geográfico } \\
\text { Militar. } \\
\text { MAPAS IGM. }\end{array}$} & \multirow{2}{*}{$\begin{array}{l}\text { INEC. División } \\
\text { Político Admi- } \\
\text { nistrativa del } \\
\text { Ecuador. }\end{array}$} & \multirow{2}{*}{$\begin{array}{l}\text { INEGI. Marco } \\
\text { geo-estadístico } \\
2010 \text { versión } 5.0 \\
\text { AGEM. }\end{array}$} \\
\hline Extensión territorial & & & & \\
\hline Extensión territorial urbana & $\begin{array}{l}\text { UNDP Brazil } \\
\text { Caracterização } \\
\text { do Município. }\end{array}$ & $\begin{array}{l}\text { Corporación } \\
\text { Nacional } \\
\text { Forestal. Sistema } \\
\text { de Información } \\
\text { Territorial. }\end{array}$ & $\begin{array}{l}\text { INEC. Archivo } \\
\text { Nacional de Da- } \\
\text { tos y Metadatos } \\
\text { Estadísticos. } \\
\text { Censo de Infor- } \\
\text { mación Ambien- } \\
\text { tal Económica. }\end{array}$ & $\begin{array}{l}\text { INEGI. } \\
\text { Información } \\
\text { Vectorial de } \\
\text { Localidades } \\
\text { Urbanas. }\end{array}$ \\
\hline Definición de primacía & $\begin{array}{l}\text { IBGE. RIDES, } \\
\text { Regiốes de } \\
\text { Influência das } \\
\text { Cidades. }\end{array}$ & \multirow{2}{*}{$\begin{array}{l}\text { INE. Ciudades, } \\
\text { pueblos, aldeas y } \\
\text { caseríos } 2002 \text {. }\end{array}$} & $\begin{array}{l}\text { Gobierno } \\
\text { de Ecuador. } \\
\text { Constitución } \\
\text { de Ecuador de } \\
2008 .\end{array}$ & \multirow{2}{*}{$\begin{array}{l}\text { CONAPO. } \\
\text { Delimitación } \\
\text { de las zonas } \\
\text { metropolitanas } \\
\text { de México. }\end{array}$} \\
\hline $\begin{array}{l}\text { Definición de área metro- } \\
\text { politana }\end{array}$ & $\begin{array}{l}\text { IBGE. Organiza- } \\
\text { ção Territorial e } \\
\text { Composição das } \\
\text { Regiôes Metro- } \\
\text { politanas. }\end{array}$ & & $\begin{array}{l}\text { SENPLADES. } \\
\text { Estratégia Terri- } \\
\text { torial Nacional. }\end{array}$ & \\
\hline
\end{tabular}


(continuación)

\begin{tabular}{|c|c|c|c|c|c|}
\hline & $\begin{array}{l}\text { ATOS A NIVEL } \\
\text { MUNICIPAL }\end{array}$ & BRASIL & CHILE & ECUADOR & MÉXICO \\
\hline \multicolumn{6}{|c|}{ Indicadores climáticos (1910-1930; 2000-2030) } \\
\hline \multicolumn{2}{|c|}{$\begin{array}{l}\text { Temperaturas medias dia- } \\
\text { rias; precipitaciones medias } \\
\text { diarias }\end{array}$} & \multicolumn{4}{|c|}{ UEA Climatic Research Unit. } \\
\hline \multicolumn{2}{|c|}{$\begin{array}{l}\text { Valores mensuales promedio } \\
\text { del Índice de Sequía de } \\
\text { Palmer }\end{array}$} & \multicolumn{4}{|c|}{ NOAA's National Center for Atmospheric Research. } \\
\hline NOTA & \multicolumn{5}{|c|}{$\begin{array}{l}\text { BRASIL, IBGE: INSTITUTO BRASILEIRO DE GEOGRAFIA E ESTATÍSTICA / CHILE, INE: INSTITUTO } \\
\text { NACIONAL DE ESTADÍSTICAS; SUBDERE: SUBSECRETARÍA DE DESARROLLO REGIONAL; } \\
\text { MIDEPLAN, MINISTERIO DE PLANIFICACIÓN Y COOPERACIÓN, ACTUAL MINISTERIO DE } \\
\text { DESARROLLO SOCIAL; MINSAL: MINISTERIO DE SALUD / ECUADOR, INEC: INSTITUTO } \\
\text { NACIONAL DE ESTADÍSTICA Y CENSOS; SENPLADES: SECRETARÍA NACIONAL DE PLANIFICACIÓN } \\
\text { Y DESARROLLO / MÉXICO, INEGI: INSTITUTO NACIONAL DE ESTADÍSTICA Y GEOGRAFÍA; } \\
\text { CONEVAL: CONSEJO NACIONAL DE EVALUACIÓN DE LA POLÍTICA DE DESARROLLO SOCIAL; } \\
\text { CONAPO: CONSEJO NACIONAL DE POBLACIÓN. }\end{array}$} \\
\hline & ELABORACIÓN PRO & & & & \\
\hline
\end{tabular}

Características demográficas. Recolectamos el número de residentes, número de residentes pobres, número de residentes urbanos, y tamaño medio de hogar por municipio, según los censos de 2000 y 2010.

Acceso a los servicios urbanos básicos. Construimos un índice de cobertura para cada municipio, con base en el censo de población y las encuestas a escala municipal. Un coeficiente de cero indica que ningún hogar tiene acceso a servicios urbanos básicos de alta calidad de distribución de agua, saneamiento o recolección de residuos. Por el contrario, un coeficiente de uno indica un municipio con cobertura universal de estos servicios. Cada uno de los tres servicios contribuye de igual manera al coeficiente de este índice, y solo consideramos el porcentaje de hogares que reciben servicios de alta calidad. En el caso de agua, son aquellos con al menos un grifo dentro de la vivienda conectado al servicio por tubería; en el de saneamiento, son los que residen en una vivienda con sistema de alcantarillado entubado; y en el de residuos, los que cuentan con recolección domiciliaria. Construimos este índice para cada municipio para cada ańo del censo disponible.

Características fiscales y económicas. Utilizamos los datos de gastos e ingresos municipales en servicios urbanos básicos para los años 2000 y 2010. El gasto municipal comprende las compensaciones a los empleados, los costos administrativos, de proveer servicios, de inversiones y de servicios de deuda pública. Los datos sobre el salario promedio se identifican como la remuneración por empleado antes de deducciones, y los ingresos municipales, como producto bruto interno per cápita. Asumimos las mismas funciones de producción Cobb-Douglas con rendimientos constantes a escala (Borcheding \& Deacon, 1972) para todos los municipios, lo cual implica que el capital es perfectamente móvil, pero no así la mano de obra. Por tanto, cada municipio tiene su propia medida de salario por unidad de trabajo, el cual incide de modo distinto en el costo de producción de los servicios urbanos básicos.

Los datos de ingresos municipales se basan en los ingresos fiscales anuales, incluyendo impuestos sobre la propiedad y transferencias intergubernamentales. Los ingresos por hogar se calculan como la suma del valor agregado bruto de la economía 
(es decir, el producto bruto interno) dividido por la población total. Para Brasil, Chile y Ecuador, ajustamos los valores monetarios según los índices nacionales de variaciones de precios. Para México, utilizamos un índice de precios de 32 estados basado en 46 ciudades de la muestra nacional de precios al consumidor del Instituto Nacional de Estadística y Geografía (Inegi). Los datos finales se expresan en us $\$ 2010$ (Inegi, 2011). En todos los casos, la armonización de los datos sigue las directrices del Manual de Finanzas Públicas del Fondo Monetario Internacional (FMI).

Indicadores urbanos. El municipio es la unidad geográfica más pequeña que podemos documentar. Utilizamos imágenes Landsat y datos de Sistemas de Información Geográfica (SIG) para identificar zonas urbanas dentro de un municipio y obtener indicadores espaciales. Además, creamos dos variables binarias, una que indica si un municipio es una ciudad cabecera, y otra si pertenece a un área metropolitana. En cada país, seguimos las definiciones nacionales de estas variables (México: Secretaría de Desarrollo Social [Sedesol], Consejo Nacional de Población [Conapo], Instituto Nacional de Estadística y Geografía [Inegi], 2010; Brasil: Instituto Brasileiro de Geografia e Estatística [IBGE], 2008; Chile: Instituto Nacional de Estadísticas [INE], 2005; Ecuador: Secretaría Nacional de Planificación y Desarrollo [Senplades], 2009). Indicadores climáticos. Utilizamos los datos mensuales de temperaturas medias diarias y de precipitaciones para el período 1910-1930, en alta definición para una malla de $0,5^{\circ} \times 0,5^{\circ}$. Estos datos son generados por la Unidad de Investigación Climática de la Universidad de East Anglia (University of East Anglia Climatic Research Unit [CRU]) (2014). Del mismo modo, obtuvimos los valores mensuales promedio del Índice de Sequía de Palmer (Palmer, 1965) y de humedad del suelo para una malla de $2,5^{\circ}$ x 2,5 del Centro Nacional de Investigación Atmosférica (National Oceanic and Atmospheric Administration [NOAA], 2014). Finalmente, recopilamos los datos climáticos para cada municipio mediante una rutina de interpolación esférica: tomamos los promedios ponderados de la climatología de diez ańos (temperatura, precipitaciones y humedad del suelo) para cada punto de una malla de $150 \mathrm{~km}$ que comienza en el centro geométrico de cada municipio (cgm), factorizado por el inverso del cuadrado de la distancia haversine entre cada punto de la grilla y este cgm.

\section{Resultados}

\section{Densidad y cobertura}

Este análisis comienza con una simple pregunta: ¿en qué municipalidades los hogares tienen más acceso a servicios de alta calidad en cuanto a agua, sanitarios y recolección de residuos? Se estima una regresión no paramétrica ponderada localmente (Fan, 1992) con un kernel de Epanechnikov para exhibir los niveles de cobertura municipales como función de la densidad urbana. Se restringe la muestra al $90 \%$ de las observaciones. La figura 1 indica que se observa una proporción mayor de la población urbana con acceso a servicios de alta calidad en áreas urbanas más densas. Más de dos tercios de las municipalidades cuyo nivel de cobertura está por debajo del $10 \%$ se encuentran en el primer cuartil de distribución de densidad de 
la población urbana (véase tabla 3). Por el contrario, más del $43 \%$ de las municipalidades que gozan de niveles de cobertura superiores al $90 \%$ están en el cuartil superior. Esta relación es consistente y se mantiene al desagregar la cobertura por tipo de servicio (figura 2), pero la cobertura de servicios sanitarios es mucho más baja que la de los otros dos, y la cobertura de agua es alta incluso en las áreas urbanas escasamente pobladas. A partir solamente de estas cifras, es imposible determinar en qué medida la densidad afecta los patrones de gasto municipal en servicios urbanos básicos, en particular su magnitud en distintas partes de la distribución. La figura 3 ilustra una regresión localmente ponderada que muestra la relación entre la densidad urbana (percentiles) y los gastos municipales en servicios urbanos básicos por habitante. Parecería que la relación tiene forma de $U$; sin embargo, debe verificarse la significancia estadística de un efecto causal de densidad urbana sobre el gasto local, dada la endogeneidad de la densidad respecto de los patrones de gasto. Se considera ahora la estrategia empírica para abordar esta cuestión.

TABLA 3 Porcentaje de hogares por municipio con acceso a servicios de agua, saneamiento y recolección de residuos de alta calidad, según nivel de cobertura y cuartil de densidad de población

\begin{tabular}{|c|c|c|c|c|}
\hline \multirow[b]{2}{*}{$\begin{array}{l}\text { HOGARES CON } \\
\text { COBERTURA }\end{array}$} & \multicolumn{4}{|c|}{ DENSIDAD DE POBLACIÓN URBANA (PERSONAS/KM²) } \\
\hline & $\begin{array}{c}\text { QI } \\
0-2.333 \\
\end{array}$ & $\begin{array}{c}\text { Q2 } \\
2.334-3.960\end{array}$ & $\begin{array}{c}Q 3 \\
3.96 I-6.378 \\
\end{array}$ & $\begin{array}{c}\text { Q4 } \\
\text { MAYOR A } 6.378\end{array}$ \\
\hline & \multicolumn{4}{|c|}{ Agua, saneamiento, y recolección de residuos } \\
\hline Menos del 10\% & 68,4 & 21,9 & 6,9 & 2,8 \\
\hline \multirow[t]{2}{*}{ Más del 90\% } & 3,7 & 15,0 & 38,0 & 43,4 \\
\hline & \multicolumn{4}{|c|}{ Agua } \\
\hline Menos del 10\% & 66,3 & 17,8 & 9,2 & 6,7 \\
\hline \multirow[t]{2}{*}{ Más del 90\% } & 16,9 & 23,4 & 28,8 & 31,0 \\
\hline & \multicolumn{4}{|l|}{ Saneamiento } \\
\hline Menos del 10\% & 59,7 & 24,5 & 10,8 & 5,0 \\
\hline \multirow[t]{2}{*}{ Más del 90\% } & 4,8 & 15,0 & 36,7 & 43,5 \\
\hline & \multicolumn{4}{|c|}{ Recolección de residuos } \\
\hline Menos del 10\% & 60,8 & 25,5 & 9,8 & 3,9 \\
\hline Más del 90\% & 10,1 & 20,8 & 36,8 & 32,3 \\
\hline
\end{tabular}

FUENTE ELABORACIÓN PROPIA 
FIGURA I | Relación entre la cobertura de servicios de agua, saneamiento, y recolección de residuos y densidad de población

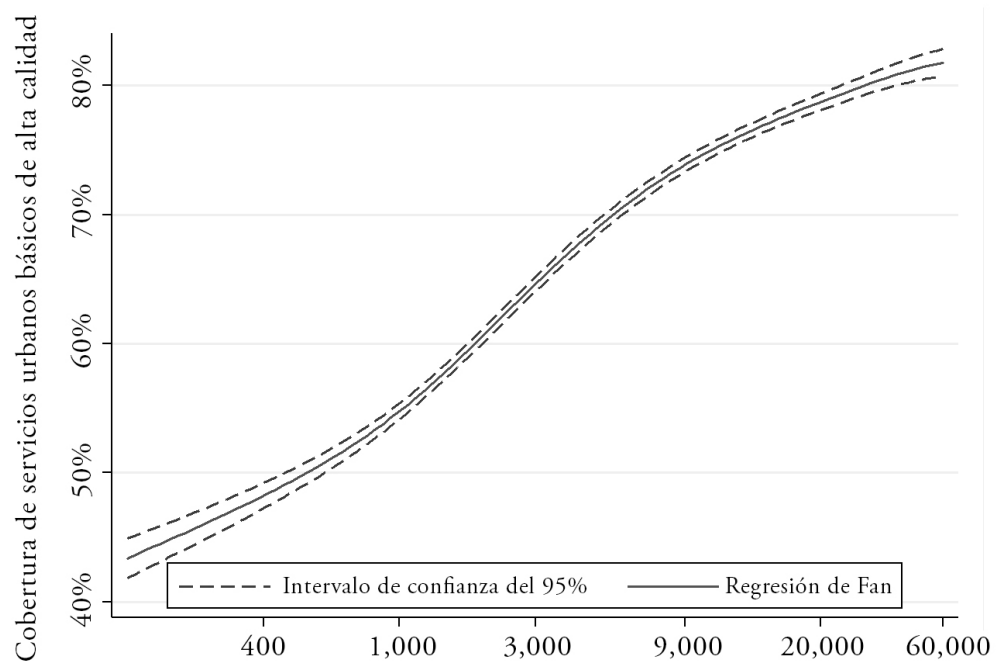

Densidad de población urbana

FUENTE ELABORACIÓN PROPIA

FIGURA 2 Relación entre cobertura de servicios de agua, saneamiento, y recolección de residuos y densidad de población, por servicio

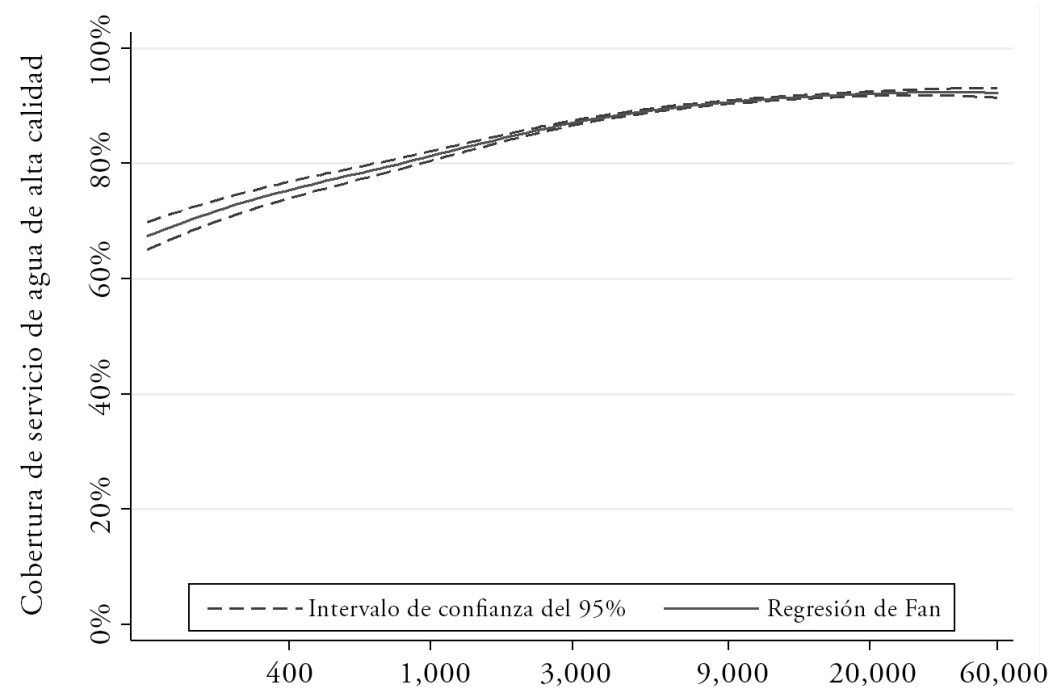

Densidad de población urbana 


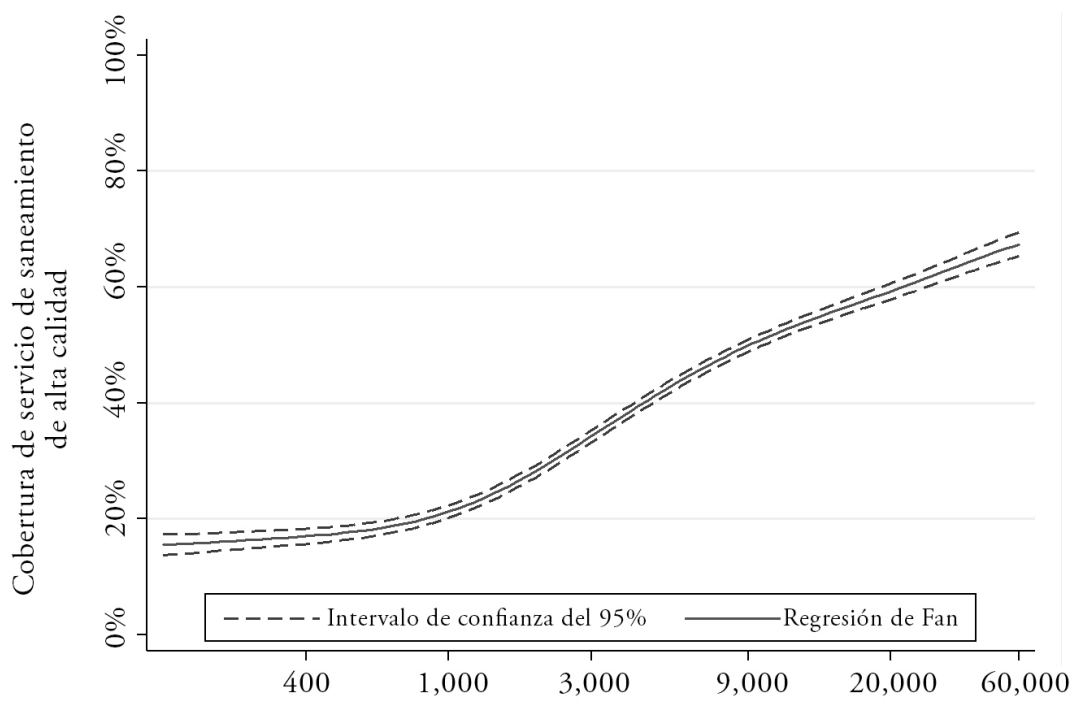

Densidad de población urbana

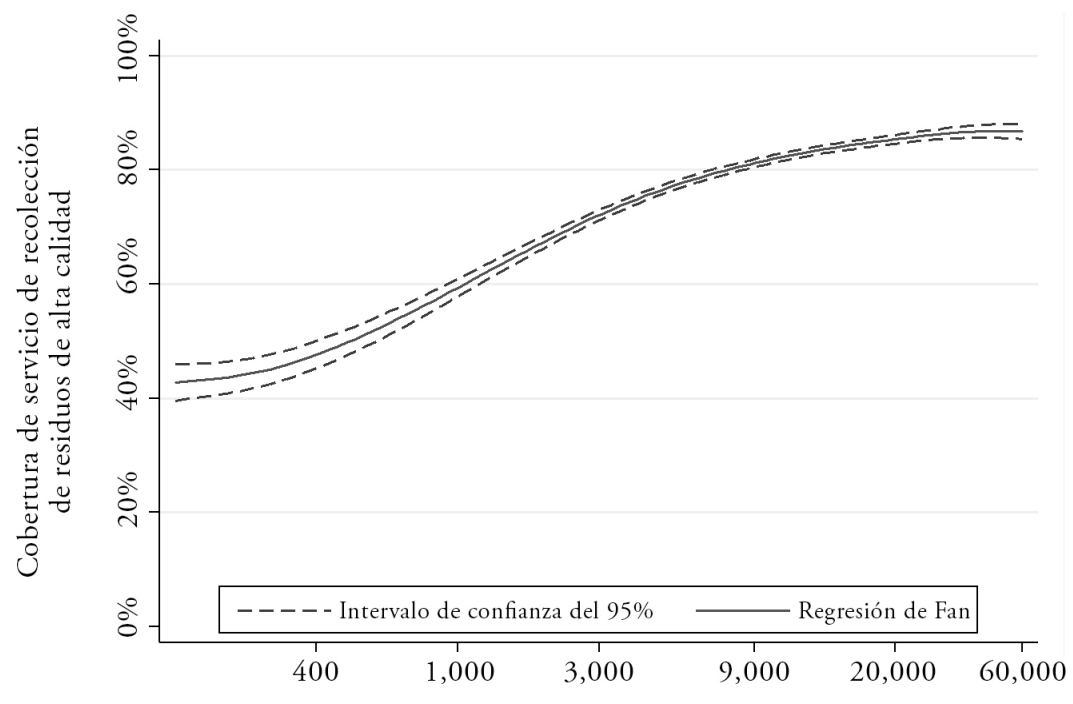

Densidad de población urbana 
FIGURA 3 | Relación entre el costo municipal de servicios de agua, saneamiento, $\mathrm{y}$ recolección de residuos por habitante y densidad de población

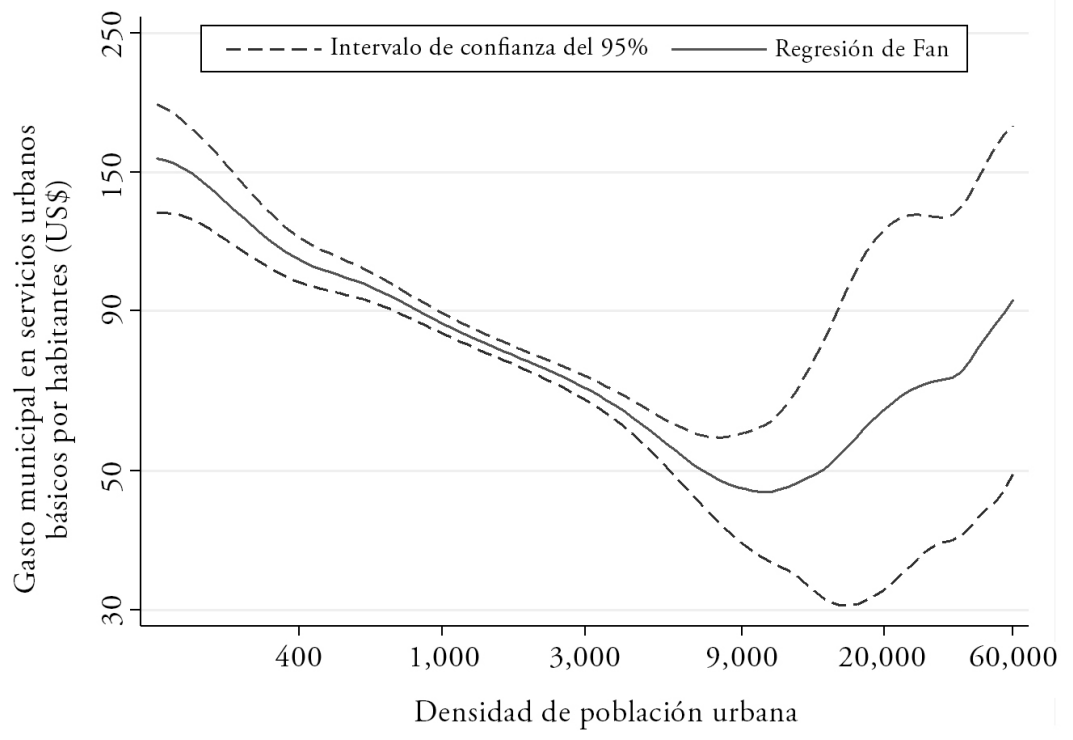

FUENTE ELABORACIÓN PROPIA

Relación de primera etapa y resultados de forma reducida

En esta sección se discute la capacidad de los instrumentos empleados en este trabajo para predecir la densidad de población corriente. Recuérdese que el conjunto de instrumentos incluye temperatura municipal retrasada, lluvia caída y condiciones de humedad del suelo. La relación de primera etapa entre este conjunto de instrumentos y la densidad de población siempre es significativa, observándose la asociación más fuerte entre humedad del suelo, temperatura y densidad urbana (tabla 4). La relación es también sólida e igualmente significativa cuando se incorporan controles para características municipales y efectos fijos bidireccionales, así como tendencias de tiempo específicas del país (regresiones 5-7, tabla 4).

TABLA 4 Datos climáticos y densidad de población (primera etapa)

\begin{tabular}{|c|c|c|c|c|c|c|c|c|}
\hline \multirow{2}{*}{$\begin{array}{c}\text { VARIABLE } \\
\text { EXPLICATIVA }\end{array}$} & \multicolumn{8}{|c|}{ MÍNIMOS CUADRADOS ORDINARIOS (OLS) } \\
\hline & $(\mathbf{I})$ & $(2)$ & (3) & $(4)$ & $(5)$ & $(6)$ & ( 7 ) & (8) \\
\hline \multirow{2}{*}{ Temperatura } & $0,0230^{* *}$ & $0,162^{* * *}$ & $0,173^{* * *}$ & $0,133^{* * *}$ & $0,0753^{* * *}$ & $0,100^{* * *}$ & $0,0701^{* * *}$ & \\
\hline & $(0,0108)$ & $(0,0411)$ & $(0,0364)$ & $(0,0333)$ & $(0,0240)$ & $(0,0279)$ & $(0,0234)$ & \\
\hline \multirow{2}{*}{$\begin{array}{l}\text { Humedad de } \\
\text { suelo }\end{array}$} & $-0,267^{* * *}$ & $0,0629^{* * *}$ & $0,0517^{* * *}$ & & $0,0289^{* * *}$ & $0,0235^{* * *}$ & & \\
\hline & $(0,0634)$ & $(0,00929)$ & $(0,00922)$ & & $(0,00577)$ & $(0,00593)$ & & \\
\hline
\end{tabular}

(continúa) 
(continuación)

\begin{tabular}{|c|c|c|c|c|c|c|c|c|}
\hline \multirow{2}{*}{$\begin{array}{c}\text { VARIABLE } \\
\text { EXPLICATIVA }\end{array}$} & \multicolumn{8}{|c|}{ MÍNIMOS CUADRADOS ORDINARIOS (OLS) } \\
\hline & (I) & (2) & (3) & (4) & (5) & (6) & (7) & (8) \\
\hline Precipitaciones & & & & $\begin{array}{l}0,0002^{*} \\
(0,0001)\end{array}$ & & & $\begin{array}{l}0,0002^{* *} \\
(0,0001)\end{array}$ & \\
\hline $\begin{array}{l}\text { Temperatura } \\
\text { futura }\end{array}$ & & & & & & & & $\begin{array}{l}0,0231 \\
(0,0187)\end{array}$ \\
\hline $\begin{array}{l}\text { Precipitaciones } \\
\text { futuras }\end{array}$ & & & & & & & & $\begin{array}{l}0,0001 \\
(0,0001)\end{array}$ \\
\hline $\begin{array}{l}\text { Prueba } F \text { de } \\
\text { instrumentos } \\
\text { excluidos }\end{array}$ & 9,05 & 23,23 & 18,92 & 14,25 & 13,26 & 10,35 & 10,02 & 1,12 \\
\hline Controles & No & No & No & No & Sí & Sí & Sí & Sí \\
\hline $\begin{array}{l}\text { Línea de } \\
\text { tendencia por } \\
\text { país }\end{array}$ & No & Sí & No & No & Sí & No & No & No \\
\hline $\begin{array}{l}\text { Efectos fijos } \\
\text { bidireccionales }\end{array}$ & No & No & Sí & Sí & Sí & Sí & Sí & Sí \\
\hline Observaciones & 13.004 & 12.030 & 12.030 & 12.812 & 10.024 & 10.024 & 10.722 & 10.722 \\
\hline$R^{2}$ & 0,0438 & 0,367 & 0,378 & 0,378 & 0,584 & 0,588 & 0,595 & 0,593 \\
\hline $\begin{array}{l}\text { Raíz del error } \\
\text { cuadrático } \\
\text { medio }\end{array}$ & 1,241 & 0,912 & 1,028 & 1,399 & 0,926 & 1,090 & 1,103 & 1,026 \\
\hline
\end{tabular}

ERrores Robustos ESTÁNDAR DE HUBER-WHite ENTRE PARÉNTESIS; ${ }^{*} P<\mathrm{O}, \mathrm{I}$ O ${ }^{* *} P<0,05{ }^{* * *} P<\mathrm{O}$, O I

FUENTE ELABORACIÓN PROPIA

Cabe notar que la relación de primera etapa continúa siendo fuerte y significativa cuando la precipitación sustituye a las condiciones de humedad del suelo como parte del conjunto de instrumentos. Si bien las pruebas estadísticas muestran que los instrumentos del clima son moderadamente fuertes (los estadísticos F oscilan entre 9,0 y 18,9), se aplica como un chequeo de identificación una especificación de experimento falsa en la cual las condiciones climáticas futuras, que deben ser ortogonales a la densidad urbana corriente, son usadas como instrumentos. Se encuentra que las estimaciones de coeficiente son sin duda estadísticamente iguales a cero (regresión 8 , tabla 4). Las temperaturas rezagadas más bajas están fuertemente asociadas con un mayor gasto municipal en las regresiones de forma reducida. Un aumento del $1 \%$ en la temperatura rezagada está asociado con una disminución del 10\% en el gasto municipal per cápita en los servicios urbanos básicos. De igual modo, un aumento del $1 \%$ en la humedad del suelo rezagada está asociado con un aumento de $0,1 \%$ en el gasto municipal en servicios urbanos básicos por cabeza (regresiones 1 y 2, tabla 5). Estas relaciones son estadísticamente significativas en el $99 \%$ de confianza. 


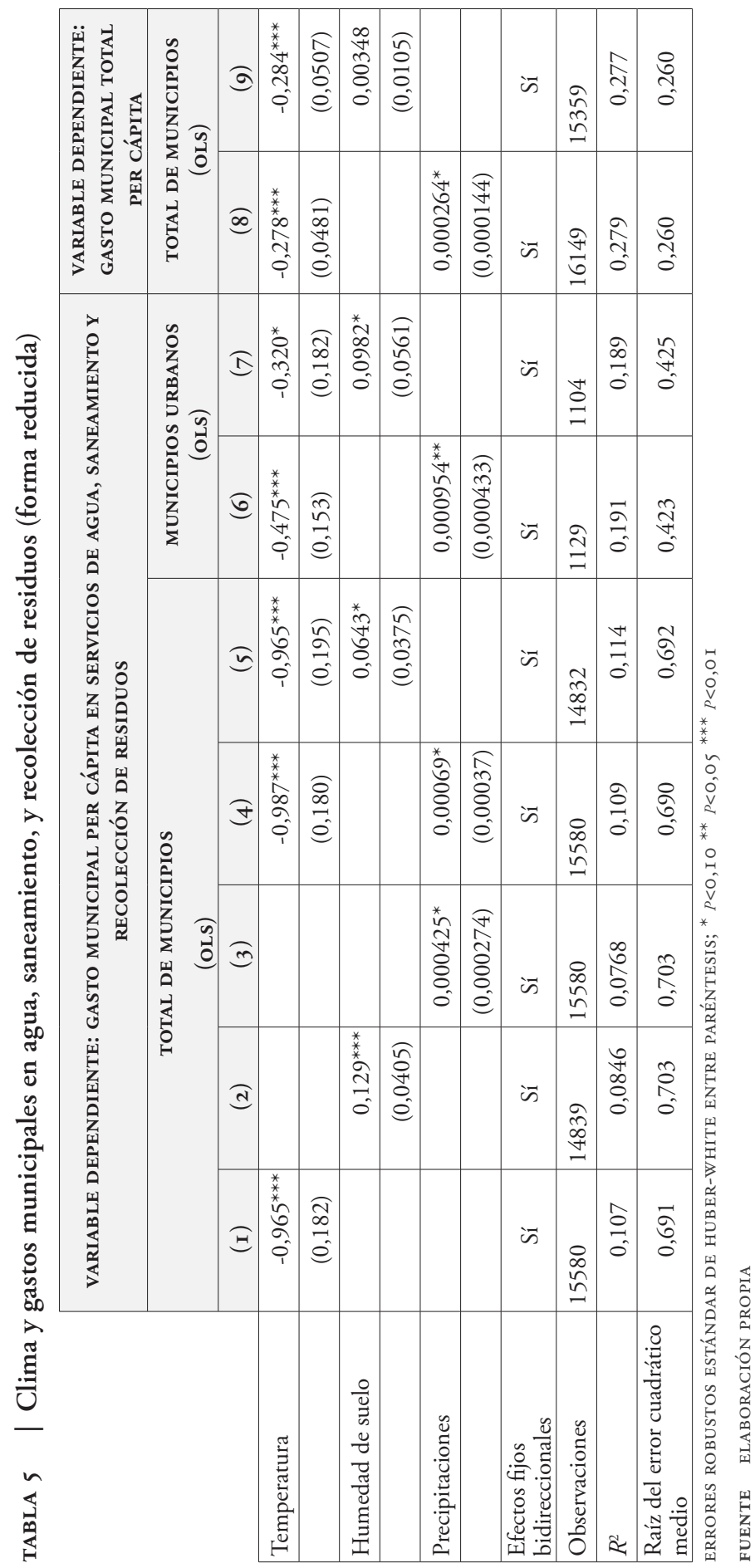


Como se esperaba, cuando solo se consideran municipalidades urbanas, las estimaciones puntuales disminuyen en magnitud, pero la relación continúa siendo estadísticamente fuerte (regresiones 6 y 7, tabla 5). De modo similar, las regresiones de forma reducida indican que los instrumentos también están asociados con el gasto municipal total por cabeza, pero a magnitudes más bajas (regresiones 8 y 9, tabla 5).

\section{Principales resultados empíricos}

Se realizan estimaciones tanto de mínimos cuadrados ordinarios (ols) como de mínimos cuadrados de dos etapas con variables instrumentales (IV-2SLS). Dada la anterior discusión teórica y análisis no paramétrico, se toman como referencia una IV-2SLS no lineal con efectos fijos municipales, tendencias en el tiempo específicas de país, y especificaciones de controles. Así, a partir de este punto el énfasis será en los resultados de esta especificación (tabla 7b, columna 10). Los resultados son similares cuando se incluyen efectos fijos temporales. Se encuentra que la relación entre densidad urbana y gasto municipal en los servicios urbanos básicos por cabeza es fuerte y en forma de $U$, sugiriendo que existe un punto de densidad óptimo (el vértice de la parábola) más allá del cual se agotan las economías de escala. Un aumento en la densidad urbana conduce a gastos de servicios urbanos básicos per cápita más bajos en áreas urbanas dispersas y de tamańo mediano, pero un mayor aumento en la densidad de la población eleva significativamente los costos de prestar servicios urbanos básicos en jurisdicciones que ya son densas. Se identifica un punto de densidad óptimo en aproximadamente 9.000 habitantes $/ \mathrm{km}^{2}$. Belém (Brasil), Santiago (Chile) y Puebla (México) se encuentran entre aquellas municipalidades cerca de la gama de densidad óptima (tabla 6). Se encuentra que la municipalidad promedio exhibe economías de escala cercanas a los 8.450 residentes, con un gasto de us $\$ 75$ por residente en la prestación de servicios urbanos básicos.

La especificación de referencia muestra que un incremento de 1 punto porcentual en la densidad de población conduce a una reducción de 0,99 puntos porcentuales en los gastos per cápita en servicios urbanos básicos. Esto es igual a una disminución en el gasto por servicio urbano básico por residente de us\$ 75 a us $\$ 67$, dado un incremento del $10 \%$ en la densidad urbana. En una municipalidad con densidades más bajas que el promedio, como las del primer cuartil (por ejemplo, 2.334 habitantes por $\mathrm{km}^{2}$ ), un incremento del $1 \%$ en la densidad urbana disminuiría el gasto per cápita en casi el 1,4\%. En cambio, en una municipalidad muy densa, como aquellas en el noveno decil (es decir, 9.659 habitantes por $\mathrm{km}^{2}$ ), un incremento del $1 \%$ en la densidad urbana lleva a un aumento en el gasto per cápita de casi el 0,1\%. Todas estas asociaciones son significativas en el nivel del 95\% (tabla 7a/b). 
TABLA 6 | Densidad de población urbana en municipalidades selectas, 2010

\begin{tabular}{|c|c|}
\hline MUNICIPALIDAD & $\begin{array}{l}\text { DENSIDAD DE POBLACIÓN URBANA } \\
\left(\text { PERSONAS } / \mathrm{KM}^{2}\right)\end{array}$ \\
\hline \multicolumn{2}{|l|}{ BRASIL } \\
\hline São Paulo & 11.047 \\
\hline Río de Janeiro & 10.928 \\
\hline Belém & 8.984 \\
\hline Brasilia & 3.544 \\
\hline \multicolumn{2}{|l|}{ CHILE } \\
\hline Santiago & 8.681 \\
\hline Valparaíso & 6.686 \\
\hline Vińa del Mar & 6.025 \\
\hline Concepción & 4.871 \\
\hline \multicolumn{2}{|l|}{ ECUADOR } \\
\hline Guayaquil & 7.082 \\
\hline Cuenca & 4.395 \\
\hline Quito & 4.059 \\
\hline Ambato & 3.340 \\
\hline \multicolumn{2}{|l|}{ MÉXICO } \\
\hline Benito Juárez (Ciudad de México) & 13.221 \\
\hline Guadalajara & 10.445 \\
\hline Puebla & 8.983 \\
\hline Monterrey & 7.678 \\
\hline
\end{tabular}

FUENTE ELABORACIÓN PROPIA

TABLA 7A | Densidad de población y gasto municipal en servicios de agua, saneamiento, y recolección de residuos, per cápita

\begin{tabular}{|c|c|c|c|c|c|}
\hline \multirow{3}{*}{$\begin{array}{c}\text { VARIABLE } \\
\text { EXPLICATIVA }\end{array}$} & \multicolumn{5}{|c|}{$\begin{array}{l}\text { VARIABLE DEPENDIENTE: GASTO MUNICIPAL EN AGUA, SANEAMIENTO } \\
\text { Y RECOLECCIÓN DE RESIDUOS PER CÁPITA }\end{array}$} \\
\hline & \multicolumn{5}{|c|}{ MÍNIMOS CUADRADOS ORDINARIOS (OLS) } \\
\hline & $(\mathbf{I})$ & (2) & (3) & (4) & $(5)$ \\
\hline \multirow{2}{*}{$\begin{array}{l}\text { Densidad de población } \\
\text { con servicios }\end{array}$} & $-0,645^{* * *}$ & $-0,774^{* * *}$ & $-0,414^{* *}$ & $-0,521^{* * *}$ & $-0,850^{* * *}$ \\
\hline & $(0,0641)$ & $(0,0742)$ & $(0,178)$ & $(0,192)$ & $(0,205)$ \\
\hline \multirow{2}{*}{$\begin{array}{l}\text { Densidad de población } \\
\text { con servicios }\end{array}$} & & & $-0,0189$ & $-0,0220$ & 0,00985 \\
\hline & & & $(0,0129)$ & $(0,0153)$ & $(0,0156)$ \\
\hline \multirow{2}{*}{ Población total } & & $-0,735^{* * *}$ & & $-0,680^{* * *}$ & $-0,823^{* * *}$ \\
\hline & & $(0,214)$ & & $(0,213)$ & $(0,216)$ \\
\hline
\end{tabular}


(continuación)

\begin{tabular}{|c|c|c|c|c|c|}
\hline \multirow{3}{*}{$\begin{array}{c}\text { VARIABLE } \\
\text { EXPLICATIVA }\end{array}$} & \multicolumn{5}{|c|}{$\begin{array}{l}\text { VARIABLE DEPENDIENTE: GASTO MUNICIPAL EN AGUA, SANEAMIENTO } \\
\text { Y RECOLECCIÓN DE RESIDUOS PER CÁPITA }\end{array}$} \\
\hline & \multicolumn{5}{|c|}{ MÍNIMOS CUADRADOS ORDINARIOS (OLS) } \\
\hline & (I) & (2) & (3) & (4) & (5) \\
\hline \multirow{2}{*}{ Población urbana } & & $0,489^{* * *}$ & & $0,538^{* * *}$ & $0,396^{* *}$ \\
\hline & & $(0,171)$ & & $(0,164)$ & $(0,197)$ \\
\hline \multirow{2}{*}{ Tamaño medio hogar } & & 0,361 & & 0,312 & 0,654 \\
\hline & & $(0,400)$ & & $(0,397)$ & $(0,409)$ \\
\hline \multirow{2}{*}{ Tasa de desempleo } & & $0,212^{* * *}$ & & $0,209^{* * *}$ & 0,0287 \\
\hline & & $(0,0307)$ & & $(0,0314)$ & $(0,0239)$ \\
\hline \multirow{2}{*}{ Tasa de pobreza } & & $-0,0313$ & & $-0,0251$ & $-0,117^{* *}$ \\
\hline & & $(0,0440)$ & & $(0,0444)$ & $(0,0516)$ \\
\hline \multirow{2}{*}{ Salario medio } & & $-0,0434$ & & $-0,0453$ & $-0,167^{* *}$ \\
\hline & & $(0,0743)$ & & $(0,0741)$ & $(0,0718)$ \\
\hline \multirow{2}{*}{ Ingresos } & & $0,517^{* * *}$ & & $0,532^{* * *}$ & $0,589^{* * *}$ \\
\hline & & $(0,159)$ & & $(0,156)$ & $(0,143)$ \\
\hline \multirow{2}{*}{ Tributos } & & $-0,341^{* * *}$ & & $-0,341^{* * *}$ & $-0,310^{* * *}$ \\
\hline & & $(0,0773)$ & & $(0,0776)$ & $(0,0711)$ \\
\hline \multirow{2}{*}{$\begin{array}{l}\text { Transferencias } \\
\text { intergubernamentales }\end{array}$} & & $0,733^{* * *}$ & & $0,732^{* * *}$ & $0,623^{* * *}$ \\
\hline & & $(0,157)$ & & $(0,158)$ & $(0,142)$ \\
\hline \multirow{2}{*}{ Área Metropolitana } & & 0,00425 & & 0,00899 & 0,0160 \\
\hline & & $(0,0806)$ & & $(0,0803)$ & $(0,0841)$ \\
\hline \multirow{2}{*}{ Cabecera urbana } & & $-0,470^{*}$ & & $-0,464^{*}$ & $-0,492^{* *}$ \\
\hline & & $(0,246)$ & & $(0,247)$ & $(0,236)$ \\
\hline \multirow{2}{*}{$\begin{array}{l}\text { Elasticidad de la } \\
\text { densidad }\end{array}$} & & & $-0,721^{* * *}$ & $-0,878^{* * *}$ & $-0,690^{* * *}$ \\
\hline & & & $(0,079)$ & $(0,105)$ & $(0,094)$ \\
\hline $\begin{array}{l}\text { Líneas de tendencia } \\
\text { por país }\end{array}$ & No & No & No & No & Sí \\
\hline $\begin{array}{l}\text { Efectos fijos } \\
\text { bidireccionales }\end{array}$ & Sí & Sí & Sí & Sí & No \\
\hline Observaciones & 13.744 & 12.172 & 13.744 & 12.172 & 12.172 \\
\hline$R^{2}$ & 0,0912 & 0,169 & 0,0920 & 0,170 & 0,201 \\
\hline $\begin{array}{l}\text { Raíz del error } \\
\text { cuadrático medio }\end{array}$ & 0,647 & 0,598 & 0,647 & 0,598 & 0,587 \\
\hline
\end{tabular}

FUENTE ELABORACIÓN PROPIA 
TABLA 7B | Densidad de población y gasto municipal en servicios de agua, saneamiento, y recolección de residuos, per cápita (continuación)

\begin{tabular}{|c|c|c|c|c|c|}
\hline \multirow{3}{*}{$\begin{array}{c}\text { VARIABLE } \\
\text { EXPLICATIVA }\end{array}$} & \multirow{2}{*}{\multicolumn{5}{|c|}{$\begin{array}{l}\text { VARIABLE DEPENDIENTE: GASTO MUNICIPAL EN AGUA, SANEAMIENTO } \\
\text { Y RECOLECCIÓN DE RESIDUOS PER CÁPITA } \\
\text { MÍNIMOS CUADRADOS EN DOS ETAPAS-VARIABLES INSTRUMENTALES } \\
\text { (IV-2SLS) }\end{array}$}} \\
\hline & & & & & \\
\hline & (6) & (7) & (8) & (9) & (Io) \\
\hline \multirow{2}{*}{$\begin{array}{l}\text { Densidad de población } \\
\text { con servicios }\end{array}$} & $2,095^{* * *}$ & $-3,561^{* * *}$ & $-12,84^{* * *}$ & $-6,982^{* * *}$ & $-9,304^{* *}$ \\
\hline & $(0,683)$ & $(1,299)$ & $(3,374)$ & $(2,238)$ & $(4,094)$ \\
\hline \multirow{2}{*}{$\begin{array}{l}\text { Densidad de pobla- } \\
\text { ción con servicios }{ }^{2}\end{array}$} & & & $0,863^{* * *}$ & $0,351^{* *}$ & \\
\hline & & & $(0,244)$ & $(0,158)$ & $(0,276)$ \\
\hline \multirow{2}{*}{ Población total } & & $-1,178^{* * *}$ & & $-2,034^{* * *}$ & $-2,643^{* *}$ \\
\hline & & $(0,357)$ & & $(0,646)$ & $(1,091)$ \\
\hline \multirow{2}{*}{ Población urbana } & & $3,735^{* *}$ & & 2,294 & $2,369^{*}$ \\
\hline & & $(1,502)$ & & $(1,491)$ & $(1,392)$ \\
\hline \multirow{2}{*}{ Tamaño medio hogar } & & $-0,0413$ & & 0,830 & 1,544 \\
\hline & & $(0,471)$ & & $(0,717)$ & $(1,143)$ \\
\hline \multirow{2}{*}{ Tasa de desempleo } & & $0,201^{* * *}$ & & $0,246^{* * *}$ & 0,0897 \\
\hline & & $(0,0358)$ & & $(0,0432)$ & $(0,0580)$ \\
\hline \multirow{2}{*}{ Tasa de pobreza } & & $0,223^{*}$ & & 0,0729 & $-0,0236$ \\
\hline & & $(0,123)$ & & $(0,124)$ & $(0,0888)$ \\
\hline \multirow{2}{*}{ Salario medio } & & 0,00144 & & 0,0110 & $-0,179$ \\
\hline & & $(0,105)$ & & $(0,110)$ & $(0,113)$ \\
\hline \multirow{2}{*}{ Ingresos } & & $0,754^{* * *}$ & & $0,471^{*}$ & $0,683^{* * *}$ \\
\hline & & $(0,249)$ & & $(0,281)$ & $(0,238)$ \\
\hline \multirow{2}{*}{ Tributos } & & $-0,391^{* * *}$ & & $-0,384^{* * *}$ & $-0,342^{* * *}$ \\
\hline & & $(0,0968)$ & & $(0,0886)$ & $(0,0866)$ \\
\hline \multirow{2}{*}{$\begin{array}{l}\text { Transferencias } \\
\text { intergubernamentales }\end{array}$} & & $0,782^{* * *}$ & & $0,796^{* * *}$ & $0,657^{* * *}$ \\
\hline & & $(0,184)$ & & $(0,169)$ & $(0,161)$ \\
\hline \multirow{2}{*}{ Área Metropolitana } & & $-0,0145$ & & $-0,0947$ & $-0,0478$ \\
\hline & & $(0,0872)$ & & $(0,0892)$ & $(0,0944)$ \\
\hline \multirow{2}{*}{ Cabecera urbana } & & $-0,574^{* *}$ & & $-0,659^{* *}$ & $-0,723^{* *}$ \\
\hline & & $(0,290)$ & & $(0,288)$ & $(0,316)$ \\
\hline
\end{tabular}


(continuación)

\begin{tabular}{|c|c|c|c|c|c|}
\hline \multirow{3}{*}{$\begin{array}{l}\text { VARIABLE } \\
\text { EXPLICATIVA }\end{array}$} & \multicolumn{5}{|c|}{$\begin{array}{c}\text { VARIABLE DEPENDIENTE: GASTO MUNICIPAL EN AGUA, SANEAMIENTO } \\
\text { Y RECOLECCIÓN DE RESIDUOS PER CÁPITA }\end{array}$} \\
\hline & \multicolumn{5}{|c|}{$\begin{array}{l}\text { MÍNIMOS CUADRADOS EN DOS ETAPAS-VARIABLES INSTRUMENTALES } \\
\text { (IV-2SLS) }\end{array}$} \\
\hline & (6) & (7) & (8) & (9) & (Iо) \\
\hline \multirow{2}{*}{$\begin{array}{l}\text { Elasticidad de la } \\
\text { densidad }\end{array}$} & & & $-1,177$ & $-1,276$ & $-0,995$ \\
\hline & & & $(1,069)$ & $(1,427)$ & $(1,194)$ \\
\hline $\begin{array}{l}\text { Líneas de tendencia } \\
\text { por país }\end{array}$ & No & No & No & No & Sí \\
\hline $\begin{array}{l}\text { Efectos fijos } \\
\text { bidireccionales }\end{array}$ & Sí & Sí & Sí & Sí & No \\
\hline Observaciones & 12.030 & 10.024 & 12.030 & 10.024 & 10.024 \\
\hline \multicolumn{6}{|l|}{$R^{2}$} \\
\hline $\begin{array}{l}\text { Raíz del error } \\
\text { cuadrático medio }\end{array}$ & 1,072 & 1,138 & 1,654 & 1,146 & 1,240 \\
\hline $\begin{array}{l}\text { Prueba de } F \text { de } \\
\text { Anderson-Rubin } \\
\text { ( } p \text {-valor) }\end{array}$ & 0,000 & 0,001 & 0,000 & 0,001 & 0,009 \\
\hline $\begin{array}{l}\text { Prueba de } X^{2} \text { de } \\
\text { Anderson-Rubin } \\
\text { ( } p \text {-valor) }\end{array}$ & 0,000 & 0,000 & 0,000 & 0,000 & 0,008 \\
\hline $\begin{array}{l}\text { Estadístico } S \text { de } \\
\text { Stock-Wright ( } p \text {-valor) }\end{array}$ & 0,000 & 0,000 & 0,000 & 0,000 & 0,010 \\
\hline $\begin{array}{l}\text { Estadístico LR de } \\
\text { Anderson ( } p \text {-valor) }\end{array}$ & 0,000 & 0,000 & 0,000 & 0,000 & 0,000 \\
\hline $\begin{array}{l}\text { Estadístico } F \text { de } \\
\text { Kleibergen-Paap }\end{array}$ & 18,92 & 10,35 & 16,70 & 9,903 & 11,18 \\
\hline $\begin{array}{l}\text { Estadístico } J \text { de } \\
\text { Hansen ( } p \text {-valor) }\end{array}$ & 0,000 & 0,018 & & & \\
\hline
\end{tabular}

ERRORES ROBUSTOS ESTÁNDAR DE HUBER-WHITE ENTRE PARÉNTESIS; ${ }^{*} P<0$, IO ${ }^{* *} P<0,05{ }^{*} * *$ P $P$, O I

FUENTE ELABORACIÓN PROPIA

El impacto de la densidad urbana en el gasto en servicios urbanos básicos por cabeza es significativo en especificaciones alternativas. Para mitigar aún más las potenciales violaciones a la restricción de exclusión (es decir, el clima debería afectar los patrones de gasto municipal solo a través de la densidad), restringimos nuestra muestra a las municipalidades urbanas. En efecto, la violación más seria a la restricción de exclusión es un potencial efecto climático sobre el ingreso. Sin embargo, si bien existe evidencia de que el clima está sumamente relacionado con el ingreso en las áreas rurales, no se ha encontrado que ejerza un claro efecto en los centros urbanos (Guerrero Compeán, 2013). Cuando se excluyen las municipalidades no urbanas, la elasticidad de la densidad de la población, de acuerdo con la especificación de referencia, es de 
aproximadamente 1,5 para la municipalidad promedio (regresión 2, tabla 8). Los resultados continúan siendo estadísticamente significativos en el nivel del 95\%.

Nuevamente, se encuentra evidencia en apoyo a una relación en forma de $U$, con municipalidades de baja (alta) densidad exhibiendo economías (deseconomías) de escala. Para municipalidades urbanas dispersamente pobladas -en el primer decil-, un incremento de 1 punto porcentual en la densidad de la población conduce a una disminución de 3,4\% en el gasto municipal per cápita en los servicios urbanos básicos. En cambio, para la municipalidad urbana en el noveno decil, un incremento de 1 punto porcentual en la densidad de la población conduce a un incremento de 0,4 puntos porcentuales en los gastos de servicios urbanos básicos por cabeza.

Los resultados de los efectos fijos IV-2SLS son robustos a una variable dependiente alternativa. Cuando se considera la relación entre densidad urbana y gasto municipal total por cabeza -en oposición al gasto per cápita en servicios urbanos básicos-, se encuentra que la mayoría de las municipalidades exhiben economías de escala, con el punto mínimo encontrándose en una densidad de población de más de 50.000 personas por kilómetro cuadrado (regresión 4, tabla 8). De igual modo, la elección de instrumentos no cambia el significado estadístico de estos resultados. La densidad de población urbana no tiene un impacto estadísticamente diferencial sobre el gasto en servicios urbanos básicos por habitante (tanto para las especificaciones agrupadas como solo urbanas) cuando se incluye la precipitación como instrumento adicional (regresiones 1 y 3 , tabla 8).

TABla 8 Densidad de población y gasto municipal-especificación alternativa

\begin{tabular}{|c|c|c|c|c|}
\hline & \multicolumn{3}{|c|}{ VARIABLE DEPENDIENTE } & VARIABLE \\
\hline & \multicolumn{3}{|c|}{$\begin{array}{l}\text { GASTO MUNICIPAL POR HABITANTE EN AGUA, } \\
\text { SANEAMIENTO Y RECOLECCIÓN DE RESIDUOS }\end{array}$} & $\begin{array}{c}\text { GASTO } \\
\text { MUNICIPAL POR } \\
\text { HABITANTE }\end{array}$ \\
\hline & \multirow{2}{*}{$\begin{array}{c}\text { TOTAL } \\
\text { MUNICIPIOS } \\
-\mathbf{I}\end{array}$} & \multicolumn{2}{|c|}{ MUNICIPIOS URBANOS } & $\begin{array}{c}\text { TOTAL } \\
\text { MUNICIPIOS }\end{array}$ \\
\hline & & -2 & -3 & -4 \\
\hline \multirow{2}{*}{$\begin{array}{l}\text { Densidad de población } \\
\text { con servicios }\end{array}$} & $-7,948^{* * *}$ & $-16,17^{* *}$ & $-21,32^{* *}$ & $-1,571^{* * *}$ \\
\hline & $(2,5960)$ & $(6,6430)$ & $(10,1000)$ & $(0,4630)$ \\
\hline \multirow{2}{*}{$\begin{array}{l}\text { Densidad de población } \\
\text { con servicios }\end{array}$} & $0,476^{* *}$ & $0,904^{* *}$ & $1,280^{* *}$ & $0,0723^{* *}$ \\
\hline & $(0,2330)$ & $(0,3960)$ & $(0,6510)$ & $(0,0340)$ \\
\hline \multirow{2}{*}{ Población total } & $-2,236^{* * *}$ & 0,0377 & 0,169 & $-0,215$ \\
\hline & $(0,7510)$ & $(0,5070)$ & $(0,5460)$ & $(0,2190)$ \\
\hline \multirow{2}{*}{ Población urbana } & 1,383 & $-0,323$ & $-1,703$ & 0,244 \\
\hline & $(2,5580)$ & $(2,2260)$ & $(1,9610)$ & $(0,3480)$ \\
\hline
\end{tabular}


(continuación)

\begin{tabular}{|c|c|c|c|c|}
\hline & & & & \\
\hline & \multicolumn{3}{|c|}{ VARIABLE DEPENDIENTE } & $\begin{array}{c}\text { VARIABLE } \\
\text { DEPENDIENTE }\end{array}$ \\
\hline & \multicolumn{3}{|c|}{$\begin{array}{l}\text { GASTO MUNICIPAL POR HABITANTE EN AGUA, } \\
\text { SANEAMIENTO Y RECOLECCIÓN DE RESIDUOS }\end{array}$} & $\begin{array}{c}\text { GASTO } \\
\text { MUNICIPAL POR } \\
\text { HABITANTE }\end{array}$ \\
\hline & \multirow{2}{*}{$\begin{array}{c}\text { TOTAL } \\
\text { MUNICIPIOS } \\
-\mathbf{I}\end{array}$} & \multicolumn{2}{|c|}{ MUNICIPIOS URBANOS } & $\begin{array}{c}\text { TOTAL } \\
\text { MUNICIPIOS }\end{array}$ \\
\hline & & -2 & -3 & -4 \\
\hline & 1,225 & 0,217 & 0,508 & $-0.598^{* * *}$ \\
\hline 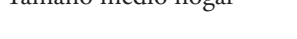 & $(0,9370)$ & $(0,7590)$ & $(0,9470)$ & $(0,2290)$ \\
\hline \multirow{2}{*}{ Tasa de desempleo } & $0,265^{* * *}$ & $0,214^{* * *}$ & $0,225^{* * *}$ & $0,0482^{* * *}$ \\
\hline & $(0,0491)$ & $(0,0498)$ & $(0,0558)$ & $(0,0095)$ \\
\hline \multirow{2}{*}{ Tasa de pobreza } & $-0,0108$ & $-0,0465$ & $-0,142$ & 0,00553 \\
\hline & $(0,2170)$ & $(0,1630)$ & $(0,1520)$ & $(0,0247)$ \\
\hline \multirow{2}{*}{ Salario medio } & 0,00753 & 0,102 & 0,139 & $-0,000996$ \\
\hline & $(0,1180)$ & $(0,1760)$ & $(0,1720)$ & $(0,0265)$ \\
\hline \multirow{2}{*}{ Ingresos } & 0,352 & 0,193 & 0,0849 & $0,351^{* * *}$ \\
\hline & $(0,3720)$ & $(0,2740)$ & $(0,2670)$ & $(0,0695)$ \\
\hline \multirow{2}{*}{ Tributos } & $-0,371^{* * *}$ & $-0,308^{* * *}$ & $-0,297^{* * *}$ & $-0,196^{* * *}$ \\
\hline & $(0,0809)$ & $(0,0888)$ & $(0,0735)$ & $(0,0234)$ \\
\hline \multirow{2}{*}{$\begin{array}{l}\text { Transferencias } \\
\text { intergubernamentales }\end{array}$} & $0,778^{* * *}$ & $0,626^{* * *}$ & $0,605^{* * *}$ & $0,387^{* * *}$ \\
\hline & $(0,1570)$ & $(0,1570)$ & $(0,1420)$ & $(0,0463)$ \\
\hline \multirow{2}{*}{ Área Metropolitana } & $-0,104$ & 0,113 & 0,0735 & 0,0199 \\
\hline & $(0,0974)$ & $(0,1380)$ & $(0,1350)$ & $(0,0247)$ \\
\hline \multirow{2}{*}{ Cabecera urbana } & $-0,669^{* *}$ & $-0,893^{* *}$ & $-0,993^{* *}$ & $-0,108^{* *}$ \\
\hline & $(0,2760)$ & $(0,4230)$ & $(0,4830)$ & $(0,0456)$ \\
\hline $\begin{array}{l}\text { Efectos fijos } \\
\text { bidireccionales }\end{array}$ & Sí & Sí & Sí & Sí \\
\hline Observaciones & 10.722 & 6.192 & 6.698 & 10.616 \\
\hline $\begin{array}{l}\text { Raíz del error cuadrático } \\
\text { medio }\end{array}$ & 1,15 & 0,988 & 1,088 & 0,24 \\
\hline $\begin{array}{l}\text { Prueba de } F \text { de Anderson- } \\
\text { Rubin ( } p \text {-valor) }\end{array}$ & 0,000 & 0,006 & 0,002 & 0,006 \\
\hline $\begin{array}{l}\text { Prueba de } X^{2} \text { de Anderson- } \\
\text { Rubin ( } p \text {-valor) }\end{array}$ & 0,000 & 0,006 & 0,002 & 0,005 \\
\hline $\begin{array}{l}\text { Estadístico } S \text { de Stock- } \\
\text { Wright ( } p \text {-valor) }\end{array}$ & 0,000 & 0,003 & 0,001 & 0,004 \\
\hline $\begin{array}{l}\text { Estadístico LR de } \\
\text { Anderson ( } p \text {-valor) }\end{array}$ & 0,034 & 0,000 & 0,001 & 0,000 \\
\hline $\begin{array}{l}\text { Estadístico } F \text { de } \\
\text { Kleibergen-Paap }\end{array}$ & 2,358 & 7,71 & 5,011 & 10,25 \\
\hline
\end{tabular}

ERRORES ROBUStOS ESTÁNDAR DE HUBER-WHITE ENTRE PARÉNTESIS; ${ }^{*} P<0$. IO ${ }^{* *} P<0.05{ }^{* * *} P<0.0$ I FUENTE ELABORACIÓN PROPIA 


\section{Conclusión e implicaciones políticas}

Las políticas actuales promueven la densidad sobre la base de que ha sido correlacionada con beneficios ambientales y en los niveles de productividad. Si bien estos son beneficios importantes, es necesario destacar que no son consecuencias automáticas de la densidad de población, sino producto de un contexto institucional y tecnológico adecuado (North, 1990). Además, este estudio, centrado en los impactos de la densidad en el gasto municipal per cápita en servicios urbanos básicos, muestra que la densidad conlleva costos específicos. Debido a que la mayor parte de las recomendaciones de políticas de densificación están dirigidas a ciudades en países en desarrollo, es de interés destacar el impacto de la densidad en aquellos municipios que tienen déficits en la provisión de servicios urbanos básicos.

Los resultados de este estudio se basan en el análisis empírico del gasto en servicios de agua entubada, saneamiento por alcantarillado y recolección domiciliaria de residuos de más de 17.000 observaciones de municipios de Brasil, Chile, Ecuador y México, tomadas de los censos de los ańos 2000 y 2010, según los cuales existe una relación significativa entre densidad de población y cobertura de servicios urbanos básicos. Más del $90 \%$ de los municipios que proveen servicios de agua, saneamiento y recolección de servicios a menos del 10\% de los hogares son territorios por debajo de la mediana de densidad de población urbana. Por el contrario, el 80\% de los municipios con niveles de cobertura por encima del $90 \%$ de los hogares está por encima de la mediana. Este mismo patrón se mantiene aun si consideramos solo a los municipios con mayoría de residentes urbanos, y cuando contamos cada servicio por separado. Cabe destacar que los resultados presentados toman el conjunto de los municipios. A la hora de implementar políticas sobre tal base, es importante considerar las características específicas de cada país. Particularmente, las posibilidades institucionales de generar planes metropolitanos de desarrollo, los cuales pueden derivar en una mejor distribución de costos y beneficios incluso con densidades menores.

Encontramos también que la relación entre densidad y gasto municipal per cápita en servicios tiene forma de $U$. La densificación contribuye a reducir gastos en los municipios de densidad baja y media, y a aumentarlos en los de alta densidad; el umbral entre estas dos condiciones es aproximadamente de 9.000 habitantes por kilómetro cuadrado. Dado que es posible que el aumento de población lleve a un municipio a incrementar su gasto en servicios, o que los hogares se muden a aquellos lugares donde los municipios invierten más en servicios, la relación entre densidad de población y gasto en servicios es endógena. Por tal motivo, utilizamos una regresión con variables instrumentales, empleando como instrumentos datos climáticos rezagados, los cuales son determinantes del crecimiento de la población, pero no del gasto municipal.

Nuestra interpretación de los resultados es que aumentar la densidad disminuye el costo per cápita en los municipios menos densos, porque contribuye a amortizar el costo de provisión de servicios; mientras que en los más densos los incrementa, porque se requiere un conjunto de tecnologías más amplio y más costoso para proporcionar cobertura de servicios a una población y un conjunto edilicio con necesidades más diversas y complejas. Además, otros factores de costos, tales como la tierra y la mano 
de obra, suelen aumentar con la densidad. Una investigación a futuro, en cuanto existan los datos adecuados, es distinguir entre los gastos de capital y los operativos, en tanto es probable que el umbral para los primeros sea mucho más denso que para los segundos. Otra importante contribución será estudiar los impactos de densidad en los costos de la prestación de servicios urbanos en asentamientos informales, dado que la prestación de servicios a posteriori es más cara.

Desde una perspectiva de política pública, la asociación entre el gasto municipal y la densidad es una buena noticia. Tenemos mejores herramientas para controlar la densidad de población que para limitar su crecimiento. Los códigos de construcción y zonificación, los incentivos fiscales, entre otros, son herramientas que pueden servir para regular las densidades. Por otra parte, es importante considerar el costo de cambiar la configuración actual cuando se planifica para la densidad. Incluso si densificar lleva a economías de escala en municipios poco poblados, puede ser muy difícil ampliar la cobertura en ellos. Por el contrario, aumentar la cobertura en un área densa puede ser más provechoso, incluso si es más caro. Esto es cierto en la medida en que los gobiernos locales puedan capitalizar fiscalmente los beneficios de una cobertura más amplia, y las áreas urbanas ya están zonificadas para una mayor densidad.

El hecho de que el gasto per cápita en servicios aumente con la densidad en municipios urbanos densos no implica que sea una mala política. Simplemente, pone en evidencia que las ciudades en crecimiento necesitan recursos también crecientes. Un estudio específico de la trama urbana y sus usos puede identificar en qué parte de un municipio tiene sentido promover la densificación, y qué forma debe tomar esta. Por ejemplo, puede ser más eficaz promover densidad en algunos barrios con un determinado conjunto de características espaciales; puede precisarse acumular tierras en zonas con baja densidad — donde debería tener un precio de mercado inferior- y luego rezonificar para densidades más altas. Además, debe incorporarse en la planificación urbana el concepto de tiempo, ya que existe un desfase entre la publicación de una nueva legislación y la implementación real de la misma. Por tal motivo, la ampliación de la calidad y cobertura de servicios urbanos básicos es también un modo de promover la densificación de la población. En ese sentido, las referencias cuantitativas de este estudio deben ser tomadas como parte de un análisis más amplio, en el que la historia, la cultura y la geografía de una ciudad deben ser consideradas. Una buena política sería preservar la diversidad dentro de cada ciudad y entre ellas.

Por último, este estudio sugiere la necesidad de un enfoque integral para la determinación de densidades ideales. El presente análisis se limita al gasto municipal en ciertos servicios, pero la densidad tiene otros costos y también otros beneficios. Entre los primeros está el impacto evidente sobre el valor de la tierra; entre los últimos, la contención de la huella ambiental urbana. La densidad como herramienta de planificación merece una consideración cuidadosa y específica del sitio. La densidad es simplemente una abstracción de una variedad de formas urbanas. Valores de densidad similares pueden ser alcanzados a través de diferentes configuraciones, y nuevas tecnologías pueden alterar las estructuras de costos de los servicios urbanos básicos actuales. Es trabajo de investigación futura comprender cómo la densidad municipal se correlaciona con las densidades a escala de la ciudad, y especialmente la articulación entre las escalas institucionales de gobierno y las de 
optimización de los costos de provisión de servicios urbanos. En todos los casos, los gobiernos necesitan adoptar una visión estratégica y de largo plazo para planificar el crecimiento urbano.

\section{Referencias bibliográficas}

Abrate G., Erbetta, F., Fraquelli, G. \& Vannoni, D. (2012). Size and density economies in refuse collection. Working Paper 274, Collegio Carlo Alberto (December). http:// www.carloalberto.org/assets/working-papers/no.274.pdf

Álvarez, I. C., Prieto, A. M. \& Zofio, J. L. (2013). Cost efficiency, urban patterns and population density when providing public infrastructure: A stochastic frontier approach. European Planning Studies, 22(6), 1235-1258. https://doi.org/10.1080/ 09654313.2013 .778957

Angel, S., Sheppard, S. C. \& Civco, D. L. (2005). The dynamics of global urban expansion. Washington, D.c.: Transport and Urban Development Department, The World Bank.

Baranzini, A. \& Faust, A. (2009). The cost structure of water utilities in Switzerland. Cabier de recherche N. ${ }^{\circ} \mathrm{HES}-\mathrm{SO} / \mathrm{HEG}-\mathrm{GE} / \mathrm{C}--10 / 5 / 1--\mathrm{CH}$. Ginebra: Centre de Recherche Appliquée en Gestion (CRAG), Haut école de gestion de Genève. https://hesso.tind.io/ record/264/files/lm.pdf

Bergstrom, T. \& Goodman, R. (1973). Private demand for public goods. American Economic Review, 63(3), 280-296.

Bollinger, C. R., Berger, M. \& Thompson, E. (2001). Smart growth and the costs of sprawl in Kentucky: Phase I \& II. Lexington, KY: University of Kentucky Center for Business and Economic Research.

Bonet, J., De la Cruz, R. \& Fretes, V. (2013). Más ingresos propios para el desarrollo local. En A. Corbacho, V. Fretes \& E. Lora (eds.), Recaudar no basta. Los impuestos como instrumento de desarrollo (Cap. 5, pp. 123-149). Washington, D.c.: Inter-American Development Bank. http://www.aecid.es/Centro-Documentacion/Documentos/ documentos\%20adjuntos/Fiscal\%20BID.pdf

Borcheding, T. \& Deacon, R. (1972). The demand for the services of non-federal governments. American Economic Review, 62(5), 891-906. http://www.gonzalo.depeco.econo.unlp. edu.ar/bspub/borcherding-deacon72.pdf

Bottasso, A. \& Conti, M. (2009). Scale economies, technology and technical change in the water industry: Evidence from the English water only sector. Regional Science and Urban Economics, 39(2), 138-147. https://doi.org/10.1016/j.regsciurbeco.2008.05.017

Bouillon, C. P. (2012). Introducción. En C. P. Bouillon (ed.), Un espacio para el desarrollo. Los mercados de vivienda en América Latina y el Caribe (pp. xxvii-xxx). Washington, D.C.: Banco Interamericano de Desarrollo. http://bit.ly/2kM3Pse

Boyko, C. T. \& Cooper, R. (2011). Clarifying and re-conceptualizing density. Progress in Planning, 76(1), 1-61. http://users.metu.edu.tr/ioguz/boyko_cooper_2011.pdf

Brakarz J., Greene, M. \& Rojas, E. (2002), Cities for all: Recent experiences with neighborhood upgrading programs. Washington, D.C.: Inter-American Development Bank. 
Burchell, R. W. \& Mukherji, S. (2003). Conventional development versus managed growth: The costs of sprawl. American Journal of Public Health, 93(9), 1534-1540. https:// www.ncbi.nlm.nih.gov/pmc/articles/PMC1448006/

Burton, E. (2000). The compact city: Just or just compact? A preliminary analysis. Urban Studies, 37(11), 1969-2006. https://doi.10.1080/00420980050162184

Cameron, S. (1989). Police cost function estimates for England and Wales. Applied Economics, 21(10), 1279-1289. https://doi.10.1080/758522324

Campbell, T. (2012). Beyond smart cities: How cities network, learn and innovate. Londres: Routledge.

Carlino, G. A., Chatterjee, S. \& Hunt, R. M. (2007). Urban density and the rate of invention. Journal of Urban Economics, 61(3), 389-419. https://doi.org/10.1016/j. jue.2006.08.003

Carruthers, J. I. \& Ulfarsson, G. F. (2003). Urban sprawl and the cost of public services. Environment and Planning B: Planning and Design, 30(4), 503-522. https:// doi.10.1068/b12847

Carruthers, J. I. \& Ulfarsson, G. F. (2008). Does 'smart growth' matter to public finance? Urban Studies, 45(9),: 1791-1823. https://doi.10.1177/0042098008093379

Ciccone, A. \& Hall, R. E. (1996). Productivity and the density of economic activity. American Economic Review, 86(1), 54-70. https://doi.10.3386/w4313

Combes P. P., Duranton G., Gobillon, L. \& Roux, S. (2010). Estimating agglomeration economies with history, geology, and worker effects. En E. L. Glaeser (ed.), Agglomeration Economics (pp. 15-66). Chicago, IL: University of Chicago Press.

Corporación Andina de Fomento (CAF). (2013). Equality and social inclusion in Latin America: Universal access to water and sanitation. Series/No. 2. Caracas: CAF.

Coyne, W. (2003). The Fiscal Cost of Sprawl. How Sprawl Contributes to Local Governments' Budget Woes. Denver, co: Environment Colorado Research and Policy Center.

Departamento Nacional de Planeación, República de Colombia. (2011). Plan Nacional de Desarrollo 2010-2014: Prosperidad para todos. Tomo II. Bogotá: Departamento Nacional de Planeación. https://colaboracion.dnp.gov.co/CDT/PND/PND20102014\%20Tomo\%20II\%20CD.pdf

Ewing, R. H. (1994). Characteristics, causes, and effects of sprawl: A literature review. Environmental and Urban Studies, 21(2), 1-15. https://doi.10.1007/978-0-38773412-5_34

Fan, J. (1992). Design-adaptive nonparametric regression. Journal of the American Statistical Association, 87(420): 998-1004. https://doi.10.2307/2290637

Feler, L. \& Henderson, J. V. (2011). Exclusionary policies in urban development: Underservicing migrant households in Brazilian cities. Journal of Urban Economics, 69(3), 253-272. https://doi.org/10.1016/j.jue.2010.09.006

Forsyth, A. (2003). Measuring density: Working definitions for residential density and building intensity. Design Brief 8. Minneapolis, MN: University of Minnesota.

Gaigne, C., Riou, S. \& Thisse, J. F. (2012). Are compact cities environmentally friendly? Journal of Urban Economics, 72(2-3), 123-136. https://doi.org/10.1016/j.jue.2012.04.001

Gilens, M. (2009). Preference gaps and inequality in representation. Political Science and Politics, 42(2), 335-341. https://doi.org/10.1017/S1049096509090441 
Glaeser, E. L. (1999). Learning in cities. Journal of Urban Economics, 46(2), 254-277. https:// doi.org/10.1006/juec.1998.2121

Glaeser, E. L. \& Gottlieb, J. D. (2009). The wealth of cities: Agglomeration economies and spatial equilibrium in the United States. Journal of Economic Literature, 47(4), 9831028. https://doi.10.3386/w14806

Glaeser, E. L., Kolko, J. \& Saiz, A. (2001), Consumer city. Journal of Economic Geography, 1(1), 27-50. https://doi.org/10.1093/jeg/1.1.27

Glaeser, E. L. \& Sacerdote, B. (1999). Why is there more crime in cities? Journal of Political Economy, 107(S6), S225-S258. https://doi.10.1086/250109

Guerrero Compean, R. (2013). Essays in climate and development. PhD dissertation, Massachusetts Institute of Technology, Cambridge, MA.

Henderson, V. (2003). The urbanization process and economic growth: The so-what question. Journal of Economic Growth, 8(1), 47-71. https://doi.10.1023/A:1022860800744

Hirsch, W. Z. (1959). Expenditure implications of metropolitan growth and consolidation. Review of Economics and Statistics, 41(3), 232-241. https://doi.10.2307/1927450

Holcombe, R. G. \& Williams, D. W. (2008). The impact of population density on municipal government expenditures. Public Finance Review, 36(3), 359-373. https:// doi.10.1177/1091142107308302

Holcombe, R. G. \& Williams, D. W. (2010). Urban sprawl and transportation externalities. The Review of Regional Studies, 40(3), 257-273. http://journal.srsa.org/ojs/index.php/ RRS/article/view/13

Hortas-Rico, M. \& Sole-Olle, A. (2010). Does urban sprawl increase the costs of providing local public services? Evidence from Spanish municipalities. Urban Studies, 47(7), 1513-1540. http://journals.sagepub.com/doi/pdf/10.1177/0042098009353620

IBI Group, Calgary. (2009). City of Calgary. The implications of alternative growth patterns on infrastructure costs. Calgary, Canadá: IBI Group. http://www.reconnectingamerica.org/ assets/Uploads/planitcalgarycoststudyanalysisaprilthird.pdf

Instituto Brasileiro de Geografia e Estatística (IBGE), Brasil. (2008). Regióes de Influência das Cidades 2007. Rio de Janeiro: IBGE. http://www.ibge.gov.br/home/geociencias/ geografia/regic.shtm?c=7

Instituto Nacional de Estadística y Geografía (Inegi), México. (2011). Documento metodológico del indice nacional de precios al consumidor. México, D.F.: Inegi. http://www.inegi.org. $\mathrm{mx} /$ est/contenidos/proyectos/inp/doc/Documento_Metodologico_INPC_INEGI.pdf

Instituto Nacional de Estadísticas (INE). (2005). Chile: Ciudades, pueblos, aldeas y caserios. Santiago, Chile: InE, Departamento de Geografía y Censos. http://www.ine.cl/canales/ usuarios/cedoc_online/censos/pdf/censo_2002_publicado_junio_2005.pdf

Inter-American Development Bank (IADB). (2013). Urban development and housing sector framework document. Washington, D.C.: IADB.

Jenks, M. \& Burgess, R. (2000). Compact cities. Sustainable urban forms for developing countries. Londres: Spon Press.

Krugman, P. R. (1991). Geography and trade. Cambridge, MA: MIT Press.

Ladd, H. F. \& Yinger, J. (1989). America's Ailing Cities: Fiscal Health and the Design of Urban Policy. Baltimore, MD: Johns Hopkins University Press.

Ladd, H. F. (1992). Population growth, density and the costs of providing public services. Urban Studies, 29(2), 273-295. https://doi.10.1080/00420989220080321 
Libertun de Duren, N. (2011). The national embeddedness of urbanization trajectories. City \& Community, 10(4), 424-430. https://doi.10.1111/j.1540-6040.2011.01384.x

London Government. (2013). Homes for London. The London Housing Strategy. Londres: Greater London Authority. https:/www.london.gov.uk/sites/default/files/london_ housing_strategy_consultation_version.pdf

México, Gobierno de la República. (2013). Plan Nacional de Desarrollo 2013-2018. México, D.F.: Gobierno de la República. http://pnd.gob.mx/

Miguel, E., Satyanath, S. \& Sergenti, E. (2004). Economic shocks and civil conflict: An instrumental variables approach. Journal of Political Economy, 112(4), 725-753. https://doi.10.1086/421174

Monterrey, Gobierno de (2011). Plan de desarrollo urbano del municipio de Monterrey 20102020. Monterrey: Secretaría de Desarrollo Urbano y Ecología.

National Oceanic \& Atmospheric Administration (NOAA) [Administración Nacional Oceánica y Atmosférica]. (2014). Palmer Drought Severity Index (PDSI) from ncar. http://www. esrl.noaa.gov/psd/data/gridded/data.pdsi.html

National People's Congress of the Popular Republic of China. (2011), China's Twelfth Five-Year Plan (2011-2015) - the full English version. http://www.britishchamber.cn/content/ chinas-twelfth-five-year-plan-2011-2015-full-english-version

Nauges, C. \& Van den Berg, C. (2007) .How "natural" are natural monopolies in the water supply and sewerage sector? Case studies from developing and transition economies. Policy Research Working Paper Series N. ${ }^{\circ}$ 4137, Washington, D.C.: The World Bank. http:// bit.ly/2kOqjw8

New York City Government. (2011). PlaNYC. A greener, greater New York. April. NYC: The City of New York.

North, D. C. (1990). Institutions, institutional change and economic performance. Cambridge, uk: Cambridge University Press.

Organisation for Economic Co-operation and Development (oECD). (2012). Compact City Policies. A comparative assessment. París: OECD. http://www.oecd.org/regional/greeningcities-regions/compact-city.htm

Palmer, W. C. (1965). Meteorological drought. Research Paper 45. Office of Climatology, u.s. Weather Bureau. Washington, D.c.: Department of Commerce. https://www.ncdc. noaa.gov/temp-and-precip/drought/docs/palmer.pdf

Pineda, C. (2005). City-county Consolidation and Diseconomies of Scale. Cambridge, MA: Harvard University.

Rapoport, A. (1977). Human aspects of urban form: Towards a man-environment approach to urban form and urban design. Nueva York: Pergamon.

Rosenthal, S. S. \& Strange, W. C. (2003). Geography, industrial organization and agglomeration. The Review of Economics and Statistics, 85(2), 377-393. https:// doi.10.1162/003465303765299882

Scott, A. J. (2006). Geography and Economy: Three lectures. Oxford: Oxford University Press.

Secretaría de Desarrollo Social (Sedesol) / Consejo Nacional de Población (Conapo) / Instituto Nacional de Estadística y Geografía (Inegi), México. (2010). Delimitación de las zonas metropolitanas de México 2010. México, D.F.: Sedesol / Conapo / Inegi. https://www. gob.mx/cms/uploads/attachment/file/112786/1_DZM_2010_PAG_1-34.pdf 
Secretaría Nacional de Planificación y Desarrollo (Senplades), Ecuador. (2009). Plan Nacional para el Buen Vivir 2009-2013. Quito: Senplades. http://www.planificacion.gob.ecl plan-nacional-para-el-buen-vivir-2009-2013/

South African National Planning Commission. (2011). National Development Plan. Vision for 2030. Johannesburgo: The Presidency of the Republic of South Africa. http://led. co.za/documents/npc-national-development-plan-vision-for-2030

Stone, B., Mednick, A. C., Holloway, T. \& Spak, S. N. (2007). Is compact growth good for air quality? Journal of the American Planning Association, 73(4), 404-418. http://dx.doi. org/10.1080/01944360708978521

un DESA (United Nations Department of Economic and Social Affairs). (2013). World Population Prospects: The 2010 Revision. http://esa.un.org/unup/unup/

un-Habitat (The United Nations Human Settlements Programme). (2012). Leveraging density: Urban patterns for a Green Economy. Nairobi: un-Habitat.

University of East Anglia Climatic Research Unit. (2014). CRU TS3.21: TS Version 3.21 (Jan. 1901 - Dec. 2012). NCAS British Atmospheric Data Centre. http://badc.nerc. ac.uk/view/badc.nerc.ac.uk__ATOM__ACTIVITY_0c08abfc-f2d5-11e2-a948$00163 \mathrm{e} 251233$ (accessed 20 March 2014).

Wheaton, W. C. \& Lewis, M. J. (2001). Urban wages and labor market agglomeration. Journal of Urban Economics, 51(3), 542-562. https://doi.org/10.1006/juec.2001.2257

Wheaton, W. C. (1998). Land use and density in cities with congestion. Journal of Urban Economics, 43(2), 258-272. https://doi.org/10.1006/juec.1997.2043

World Bank. (2014a). Urban China: Toward efficient, inclusive, and sustainable urbanization. Washington, D.c.: The World Bank. https://openknowledge.worldbank.org/ handle/10986/18865

World Bank. (2014b). World Development Indicators. http://databank.worldbank.org 